University of Nebraska - Lincoln

DigitalCommons@University of Nebraska - Lincoln

\title{
Conduction in rectangular plates with boundary temperatures specified
}

James V. Beck

Michigan State University

Neil T. Wright

Michigan State University

A. Haji-Sheikh

University of Texas, Arlington

Kevin D. Cole

University of Nebraska-Lincoln, kcole1@unl.edu

Donald E. Amos

Sandia National Laboratories, Albuquerque, NM

Follow this and additional works at: https://digitalcommons.unl.edu/mechengfacpub

Part of the Mechanical Engineering Commons

Beck, James V.; Wright, Neil T.; Haji-Sheikh, A.; Cole, Kevin D.; and Amos, Donald E., "Conduction in rectangular plates with boundary temperatures specified" (2008). Mechanical \& Materials Engineering Faculty Publications. 37.

https://digitalcommons.unl.edu/mechengfacpub/37

This Article is brought to you for free and open access by the Mechanical \& Materials Engineering, Department of at DigitalCommons@University of Nebraska - Lincoln. It has been accepted for inclusion in Mechanical \& Materials Engineering Faculty Publications by an authorized administrator of DigitalCommons@University of Nebraska Lincoln. 


\title{
Conduction in rectangular plates with boundary temperatures specified
}

\author{
James V. Beck ${ }^{\mathrm{a}, *, 1}$, Neil T. Wright ${ }^{\mathrm{a}}$, A. Haji-Sheikh ${ }^{\mathrm{b}}$, Kevin D. Cole ${ }^{\mathrm{c}}$, Donald E. Amos ${ }^{\mathrm{d}}$ \\ ${ }^{a}$ Department of Mechanical Engineering, 2555 Engineering Building, Michigan State University, East Lansing, MI 48824-1226, USA \\ ${ }^{\mathrm{b}}$ Department of Mechanical and Aerospace Engineering, The University of Texas, Arlington, TX 76019-0023, USA \\ ${ }^{\mathrm{c}}$ Department of Mechanical Engineering, N104 Scott Engineering Center, University of Nebraska-Lincoln, NE 68588-0656, USA \\ ${ }^{\mathrm{d}}$ Sandia National Laboratories, Albuquerque, NM 87185, USA
}

Received 15 November 2007; received in revised form 21 February 2008

Available online 13 April 2008

\begin{abstract}
Steady-state components of heat conduction solutions may have very slowly convergent series for temperatures and non-convergent heat fluxes for temperature boundary conditions. Previous papers have proposed methods to remove these convergence problems. However, even more effective procedures based on insights of Morse and Feshbach are given herein. In some cases it is possible to replace poorly-convergent or non-convergent series by closed-form algebraic solutions. Examples are given.
\end{abstract}

(C) 2008 Elsevier Ltd. All rights reserved.

Keywords: Heat conduction; Analytical solutions; Improved convergence; Plates

\section{Introduction}

Exact transient heat conduction solutions have many uses including verification of numerical solutions [1-4], facilitating insight, and providing building blocks for the unsteady surface element method [5, Chapter 12]. These solutions are frequently the sum of steady-state and complementary transient components $[2,3]$. The complementary transient components have rapid exponential convergence, provided the dimensionless time is sufficiently large. However, exact conduction steady-state solutions for the temperature in a rectangle with prescribed temperature boundary conditions typically converge slowly at the surface with a non-homogeneous boundary condition [6]. The problem is acute for determining the heat flux at that surface [7]. Solutions based on the separation of variables method, which we relate to the long cotime Green's function (with cotime defined as $u=t-\tau$ ), are particularly

\footnotetext{
* Corresponding author. Tel.: +1 517349 6688; fax: +1 5173531750 .

E-mail address: beck@msu.edu (J.V. Beck).

${ }^{1}$ Prof. Em.
}

prone to this problem; the series for the heat flux normal to the surface may even fail to converge at that surface.

Cole, Yen, and Crittenden [6,7] have clearly identified this problem and have proposed solutions. They have shown that there are multiple forms of the Green's function that may be used for $2 \mathrm{D}$ and $3 \mathrm{D}$ problems. Choosing the appropriate form can reduce considerably the number of terms in the summations. Heat conduction textbooks commonly recommend that the eigenvalues in multi-dimensional steady-state problems be chosen to be in the direction of the homogeneous boundary conditions. See Ref. [8] for more discussion of this point. However, this recommendation is not usually the best one [6-8]. The convergence is usually improved by using eigenfunctions in a direction perpendicular to the nonhomogeneous surface [7].

In this paper, a more nuanced approach is given that is better for prescribed temperature boundary conditions; the preferred eigenfunction direction depends upon the aspect ratio. As noted in [6-8], the series may contain a term that converges very poorly. These authors propose alternative Green's functions to improve convergence; the present 


\section{Nomenclature}

a dimensionless value of isotherm

c.t. denotes complementary transient component, Eq. (6)

$C_{n} \quad$ modified eigenvalue, $\left(=\eta_{n} \widetilde{L}\right.$, first appears in Eq. (8))

$G_{X I J}, G_{Y I J}$ Green's functions for rectangular plate with $I$ and $J$ boundary condition kinds, $\mathrm{m}^{-1}$

$I, J \quad$ indication of boundary condition kinds, e.g., 1 1st kind, 2 - 2nd kind, 3 - 3rd kind

$k$ thermal conductivity, $\mathrm{W} /(\mathrm{m} \mathrm{K})$

$K_{\max } \quad$ negative of natural logarithm of error

$L \quad$ thickness of slab, m

$\widetilde{L} \quad$ aspect ratio of the slab, $(=L / W)$

$m \quad$ summation index

$N_{m} \quad m$ th norm of $X_{m}(x)$

$n \quad$ summation index

$n_{\max }$ maximum number of terms needed for a specified error

$q_{x}, q_{y}$ heat flux at the boundary planes, $\mathrm{W} / \mathrm{m}^{2}$ $t \quad$ time, $\mathrm{s}$

$T$ temperature, ${ }^{\circ} \mathrm{C}$

$T_{0} \quad$ temperature at $x=0,{ }^{\circ} \mathrm{C}$

$u \quad$ cotime $(=t-\tau), \mathrm{s}$

$W \quad$ width of slab, $\mathrm{m}$

$X_{X I J, m}(x) m$ th eigenfunction

$x \quad$ length, $\mathrm{m}$

$x_{\mathrm{p}}, y_{\mathrm{p}}$ penetration lengths of the temperature signal, $\mathrm{m}$

$\tilde{x} \quad$ scaled length $(=x / L)$

$y \quad$ width, $\mathrm{m}$

$\tilde{y} \quad$ scaled width $(=y / W)$

Greek symbols

$\alpha \quad$ thermal diffusivity, $\mathrm{m}^{2} / \mathrm{s}$

$\beta_{m} \quad$ eigenvalue in $x$-direction $(m \pi)$

$\varepsilon \quad$ error

$\eta_{n} \quad$ eigenvalue in $y$-direction

$\tau \quad$ variable of integration, $\mathrm{s}$ paper removes this difficult term in a similar way. Beyond this poorly-convergent term, however, another term is present which impedes convergence. The removal of this term is discussed next.

Over 50 years ago Morse and Feshbach [9] gave an algebraic expression for the classical series solution that diverges at the boundary. The derivation is not transparent and the result is not widely known, although it is given (without reference) by Gebhart [10, p. 98] and Polyanin [11, p. 470]; see also Ingersoll et al. [12, pp. 34 and 307]. Primary motivations for this paper are to use and extend the Morse-Feshbach relation and also to elucidate some of its subtle steps. The problem treated herein is a classic one for a rectangle with a constant non-zero temperature at $x=0$ and zero temperatures on the three surfaces at $x=L, y=0$ and $W$. One limiting case is for a semi-infinite strip in the $x$ direction, that is $L \rightarrow \infty$; this case is treated in [9] and is denoted in this paper as $X 10 B 1 Y 11 B 00$. (The notation is described below.) Another limiting case is for a semi-infinite strip in the $y$-direction, or $W \rightarrow \infty$ (denoted as $X 11 B 10 Y 10 B 0)$; a new algebraic identity for the related summation is derived herein for this case. The method is applied here to 2D problems with temperature boundary conditions, but it can be extended to other problems, including 3D cases.

The algebraic solutions (partially algebraic, in some cases) are convenient and aid in providing insight in several ways. First, they remove the convergence problem associated with the series solution for the heat flux at the heated boundary. That can prove helpful in verification of numerical solutions. Second, algebraic forms are more insightful than series solutions. For example, the algebraic expressions can be readily used to determine the penetration dis- tance of the non-homogenous condition at $x=0$. Third, the solutions may be helpful in experimentation by providing quickly evaluated expressions for parameter estimation. Fourth, these steady-state solutions may be part of several different transient solutions and several variations of the basic problem are possible. The solutions can be utilized in the unsteady surface element method [5, Chap. 12].

Other unrelated methods are available to improve convergence of series in exact heat conduction solutions. The time-partitioning method [2-5] is a powerful and general method, but numerical integration is used in [4] and large aspect ratios may cause difficulties. This paper incorporates some of the ideas in [6] and extends them by removing some slowly-convergent series for prescribed temperatures replacing them with two algebraic forms, which do not have convergence problems. The first of these algebraic forms is given by Morse and Feshbach [9] and the second is believed to an original contribution. These two algebraic forms are particularly powerful for the large and small (compared to one) aspect ratios of $L$ divided by $W$.

Another method to obtain the steady-state component uses the complementary transient component [8] with the advantage of its exponential convergence. It is useful for locations away from the non-homogeneous surface since for sufficiently small dimensionless time it is the negative of the steady-state solution. Herein however, the sum of an algebraic term (which removes the slowly converging component) and the remaining series is simpler and is not restricted to particular regions in the rectangle.

The work of Melnikov [13,14] is important because it introduces some algebraic 2D steady-state Green's functions. Duffy [15] also gives steady Green's functions for several 2D geometries including the rectangle and semi-infinite 
slab. Significant contributions have been made by de Monte [16,17] and Haji-Sheikh et al. [18-20] for transient and multidimensional heat conduction problems.

In order to be efficient in the description of multiple problems related to the rectangle problem, the heat conduction number system in [5] is used. A brief description is now given. Three common heat conduction boundary conditions are prescribed temperature, prescribed heat flux, and prescribed ambient temperature. For simplicity these are termed boundary conditions of the 1st, 2nd, and 3rd kinds and are used in the number system. Another kind, called the 0th kind, is for a boundary at infinity in the $x$ or $y$-direction. A plate with a temperature prescribed at $x=0$ and another temperature at $x=L$ is denoted $X 11$. The $X$ denotes the $x$-direction; the first " 1 " denotes a boundary condition of the first kind at $x=0$ and the second " 1 " denotes the 1st kind boundary condition at $x=L$. Boundary condition modifiers, denoted by " $B$," are used to describe a boundary condition, with a " 1 " denoting a constant in both space and time and a " 0 " denoting a zero value of the boundary temperature $T$, heat flux $q$ or the ambient temperature; a zero value at a bound- ary is commonly termed a homogeneous condition. For the rectangular problem described above, the steady-state notation is $X 11 B 10 Y 11 B 00$; see Fig. 1a. If the problem is transient with an initial temperature distribution of zero, this notation is modified by adding " $T 0$ " at the end.

\section{Problem}

A transient version of the problem can be mathematically described by

$$
\begin{aligned}
& \frac{\partial^{2} T}{\partial x^{2}}+\frac{\partial^{2} T}{\partial y^{2}}=\frac{1}{\alpha} \frac{\partial T}{\partial t}, \quad 0<x<L, 0<y<W, t>0 \\
& T(0, y, t)=T_{0}, T(L, y, t)=0 \\
& T(x, 0, t)=0, T(x, W, t)=0 \\
& T(x, y, 0)=0
\end{aligned}
$$

The body is a rectangle with dimensions $L$ and $W$ in the $x$ and $y$-dimensions, respectively. See Fig. 1a. The thermal diffusivity $\alpha$ is the only property present in this temperature problem and it is independent of location and temperature. The temperature is $T_{0}$ at $x=0$ and zero at the other three

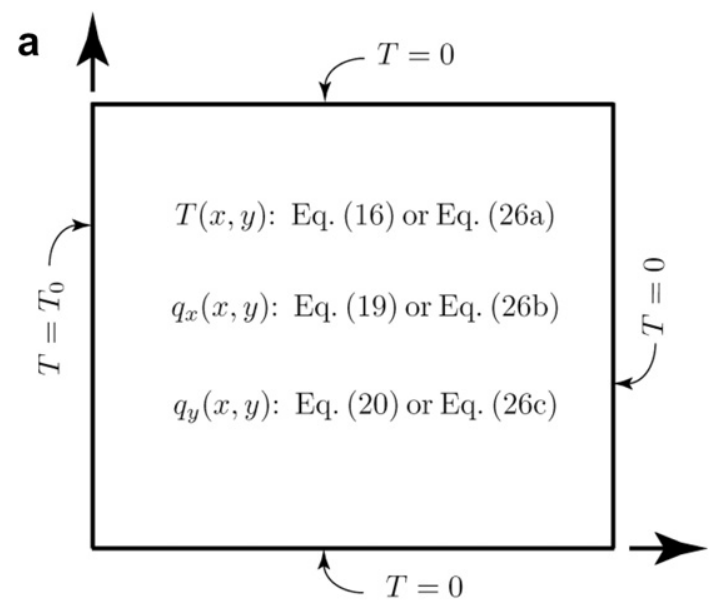

X11B10Y11B00

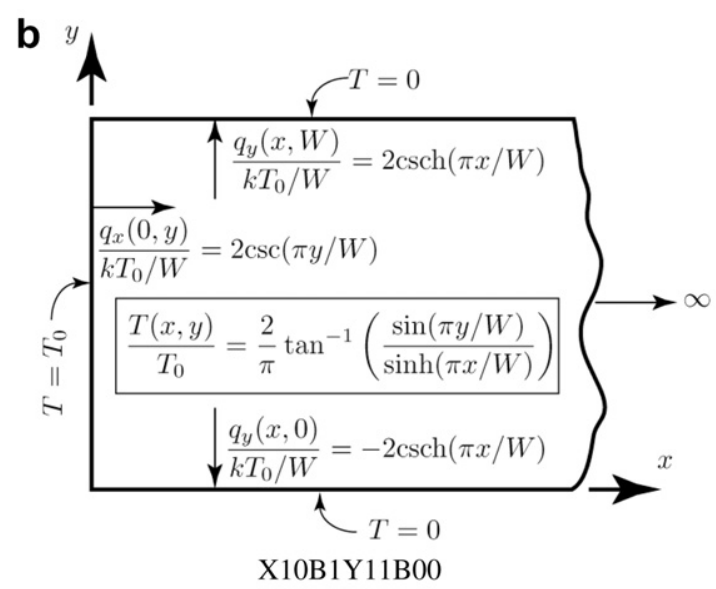

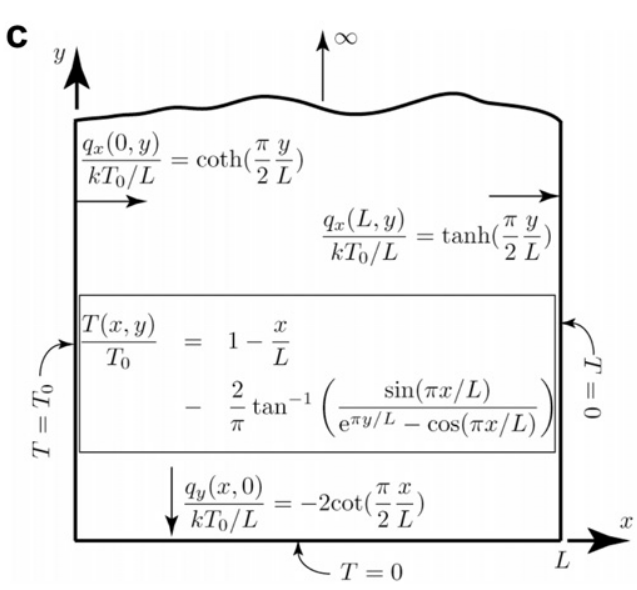

X11B10Y10B0

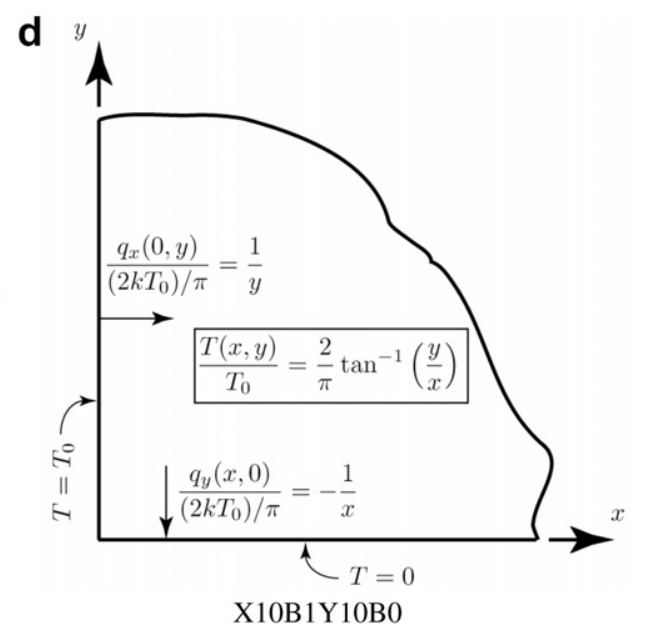

Fig. 1. Basic geometry for rectangular body, $L$ by $W$, with a temperature of $T_{0}$ and $x=0$ and $T=0$ at the other surfaces shown in Fig. 1 a. Fig. $1 \mathrm{~b}$ is for the limiting case of $L \rightarrow \infty$. Fig. 1c is for the limiting case of $W \rightarrow \infty$ and Fig. 1d is for both $L \rightarrow \infty$ and $W \rightarrow \infty$. 
surfaces; the initial temperature is zero. The notation for this problem as given in [5] is $X 11 B 10 Y 11 B 00 T 0$. It is a classic problem and has been considered in many books. As mentioned above and explained below, many of these solutions do not satisfactorily converge, particularly for the $x$-direction heat flux at $x=0$.

The problem described by Eqs. (1)-(3) is investigated in [7] and substantial improvements are given compared to the solutions commonly given in textbooks. The methods herein present further improvement and even eliminate summations for certain aspect ratios and accuracies.

The solution can be obtained several ways, one of which uses steady-state Green's functions [6,7,21,22]. Another method, and the one used here, is the long cotime form of the Green's functions; this solution has similarities with the classical separation of variables solution. The Green's function solution $[5$, p. 51] is

$$
\begin{aligned}
T(x, y, t)= & T_{0} \alpha \int_{u=0}^{t}\left(-\frac{\partial G_{X 11}}{\partial n^{\prime}}(x, 0, u)\right) \\
& \times \int_{y^{\prime}=0}^{W} G_{Y 11}\left(y, y^{\prime}, u\right) \mathrm{d} y^{\prime} \mathrm{d} u
\end{aligned}
$$

where [5, p. 482] (with $n^{\prime}=-x^{\prime}$ )

$G_{X 11}\left(x, x^{\prime}, u\right)=\frac{2}{L} \sum_{m=1}^{\infty} \sin (m \pi \tilde{x}) \sin \left(m \pi \tilde{x}^{\prime}\right) \mathrm{e}^{-\left(\frac{m \pi}{L}\right)^{2} \alpha u}$,

$\tilde{x} \equiv \frac{x}{L}$

$-\frac{\partial G_{X 11}}{\partial n^{\prime}}(x, 0, u)=\frac{2}{L^{2}} \sum_{m=1}^{\infty} \sin (m \pi \tilde{x}) m \pi \mathrm{e}^{-\left(\frac{m \pi}{L}\right)^{2} \alpha u}$

$\int_{y^{\prime}=0}^{W} G_{Y 11}\left(y, y^{\prime}, u\right) \mathrm{d} y^{\prime}=4 \sum_{n=1}^{\infty} \frac{\sin \left(\eta_{n} \tilde{y}\right)}{\eta_{n}} \mathrm{e}^{-\left(\frac{\eta_{n}}{W}\right)^{2} \alpha u}$

$\tilde{y} \equiv \frac{y}{W}, \quad \eta_{n}=(2 n-1) \pi$

Using Eqs. (5b) and (5c) in Eq. (4), the solution can be written in two parts in the form

$T(x, y, t)=T_{\text {c.t. }}(x, y, t)+T(x, y)$

where the first term on the right is the "complementary transient" and the second is the steady-state component $[2,3]$. In explicit form these two solution components are

$$
\begin{aligned}
T_{\text {c.t. }}(x, y, t)= & -T_{0} 8 \sum_{m=1}^{\infty} \sum_{n=1}^{\infty} \\
& \times \frac{m \pi \sin (m \pi \tilde{x}) \sin \left(\eta_{n} \tilde{y}\right)}{\eta_{n}\left[(m \pi)^{2}+\left(\eta_{n} \widetilde{L}\right)^{2}\right]} \mathrm{e}^{-\left[(m \pi)^{2}+((2 n-1) \widetilde{L})^{2}\right] \frac{g t}{L^{2}}} \\
T(x, y)= & T_{0} 8 \sum_{m=1}^{\infty} \sum_{n=1}^{\infty} \frac{m \pi \sin (m \pi \tilde{x}) \sin \left(\eta_{n} \tilde{y}\right)}{\eta_{n}\left[(m \pi)^{2}+\left(\eta_{n} \tilde{L}\right)^{2}\right]} \\
= & T_{0} 4 \sum_{n=1}^{\infty} \frac{\sin \left(\eta_{n} \tilde{y}\right)}{\eta_{n}} 2 \sum_{m=1}^{\infty} \frac{m \pi \sin (m \pi \tilde{x})}{\left[(m \pi)^{2}+\left(\eta_{n} \widetilde{L}\right)^{2}\right]} \\
\widetilde{L} & \equiv \frac{L}{W}
\end{aligned}
$$

When the time $t$ is zero, observe that the complementary transient is equal to the negative of the steady-state solution [3]. Eq. (7a) can be computed in an efficient manner since it has exponential convergence, provided $t \neq 0$; the terms in the exponent of the exponential term increase as the square of the eigenvalues which further speeds convergence. The steady-state term in Eq. (7b) should not be evaluated directly because it is a slowly-convergent doublesummation. It is possible to remove either the $m$ or $n$ series to obtain a single sum. The relative convergence of the $m$ and $n$ series depends upon the aspect ratio $\widetilde{L}$. Hence, to cover the complete domain, two different single-sum series are needed. They are generally exponentially converging series. However, certain components of the series might cause slow convergence, but using some summation identities these components can be replaced by algebraic expressions.

\section{Standard solution (eigenvalues in the homogeneous direction)}

The most common exact solution for the steady-state component of Eq. (6) uses the separation of variables method with the eigenvalues in the homogeneous direction ( $y$ in this problem). It is also obtained using an identity denoted $X F 11 B 10$ in [8, p. 267],

$$
\begin{aligned}
& 2 \sum_{m=1}^{\infty} \frac{m \pi \sin (m \pi \tilde{x})}{(m \pi)^{2}+C_{n}^{2}}=\frac{\sinh \left[C_{n}(1-\tilde{x})\right]}{\sinh \left[C_{n}\right]}=\frac{\mathrm{e}^{-C_{n} \tilde{x}}-\mathrm{e}^{-C_{n}(2-\tilde{x})}}{1-\mathrm{e}^{-2 C_{n}}}, \\
& C_{n} \equiv \eta_{n} \widetilde{L}
\end{aligned}
$$

Either way, this typical steady-state expression is given by (and denoted $X 11 B 10 Y 11 B 00$ )

$$
\begin{aligned}
\frac{T(\tilde{x}, \tilde{y})}{T_{0}} & =4 \sum_{n=1}^{\infty} \frac{\sin \left(\eta_{n} \tilde{y}\right)}{\eta_{n}} \frac{\sinh \left[\eta_{n} \widetilde{L}(1-\hat{x})\right]}{\sinh \left[\eta_{n} \widetilde{L}\right]} \\
& =4 \sum_{n=1}^{\infty} \frac{\sin \left(\eta_{n} \tilde{y}\right)}{\eta_{n}} \frac{\mathrm{e}^{-\eta_{n} \tilde{L} \tilde{x}}-\mathrm{e}^{-\eta_{n} \tilde{L}(2-\tilde{x})}}{1-\mathrm{e}^{-2 \eta_{n} \widetilde{L}}}
\end{aligned}
$$

The number of required terms to obtain an error of $\varepsilon$ can be obtained by using $\varepsilon=\exp \left(-K_{\max }\right)$. For $\varepsilon=0.01, K_{\max }=$ 4.61; for $\varepsilon=0.00001, K_{\max }=11.5$; and for $\varepsilon=10^{-10}, K_{\max }$ $=23$. (see Section 7 for more discussion.) Then, the required number of terms for Eq. (9a) is

$n_{\max }=\operatorname{ceil}\left(\frac{K_{\max }}{2 \pi} \frac{W}{x}\right)$

where "ceil" denotes the integer part plus 1. (Usually Eq. (9a) indicates more terms than are necessary in the series so it is conservative.) Eq. (9a) has exponential convergence at all $x$-values except $x=0$, at which point it converges very slowly. Unfortunately, the $T=T_{0}$ boundary, which is at $x=0$, is the most important one. Expressions similar to Eq. (9a) are given in Carslaw and Jaeger [23, p. 167], Gebhart [10, p. 101] and Arpaci [24, pp. 215, 216]; Myers $[25$, p. 129] is the same if $W=L=1$. 
For $L \rightarrow \infty$, Eq. (9a) can be written as

$\frac{T_{X 10 B 1 Y 11 B 00}(x, y)}{T_{0}}=4 \sum_{n=1}^{\infty} \frac{\sin \left(\eta_{n} \tilde{y}\right)}{\eta_{n}} \mathrm{e}^{-\eta_{n} x / W}, \quad L \rightarrow \infty$

This solution, denoted $X 10 B 1 Y 11 B 00$ and depicted in Fig. 1 b, is the dominant term in Eq. (9a). Eq. (9c) is similar to that given by Ozisik [26, p. 83] and Arpaci [24, p. 197]; it converges slowly as $x=0$ is approached.

Since Eq. (9c) is the slowly convergent component in Eq. (9a), examining Eq. (9c) yields insight into the complete solution for the $X 11 B 10 Y 11 B 00$ problem. For that reason, results obtained from Eq. (9c) are examined further. Formally, the heat flux in the $x$-direction is

$\frac{q_{x, X 10 B 1 Y 11 B 00} W}{k T_{0}}(0, y)=4 \sum_{n=1}^{\infty} \sin \left(\eta_{n} \tilde{y}\right), \quad L \rightarrow \infty$

This series does not converge, which is unsatisfactory. The heat flux in the $y$-direction at $y=0$ is

$\frac{q_{y, X 10 B 1 Y 11 B 00} W}{k T_{0}}(x, 0)=-4 \sum_{n=1}^{\infty} \mathrm{e}^{-\eta_{n} x / W}, \quad L \rightarrow \infty$

This flux converges exponentially provided $x \neq 0$, and thus is superior to Eq. (10a). However, algebraic expressions given in Section 4 for the temperature and heat fluxes are preferable.

\section{Improved standard solution (eigenvalues in the homogeneous direction)}

The two equations, Eqs. (9a) and (9c), do not have efficient convergence properties at the important location of $x=0$. Discussed in Section 4.1 are the improvements afforded by the Morse and Feshbach [9, p. 1179] summation identity for the plate being semi-infinite in the $x$ direction. Section 4.2 provides results for a finite body (X11B10Y11B00), also using the same identity.

\subsection{Solution for the semi-infinite body (X10B1Y11B00)}

The temperature and heat flux expressions in this subsection are for a semi-infinite plate. The identity given by Morse and Feshbach is derived in a more direct manner in Appendix A than it is in [9]. Interchanging $x$ and $y$ and also $L$ and $W$ in Eq. (A.1) gives an alternative expression for Eq. (9c). The solution, which is computationally appropriate for "large" $L / W$ (and denoted $X 10 B 1 Y 11 B 00$ ), is

$$
\begin{aligned}
\frac{T_{X 10 B 1 Y 11 B 00}(x, y)}{T_{0}} & =4 \sum_{n=1}^{\infty} \frac{\sin \left(\eta_{n} \tilde{y}\right)}{\eta_{n}} \mathrm{e}^{-\eta_{n} \widetilde{L} \tilde{x}} \\
& =\frac{2}{\pi} \tan ^{-1}\left[\frac{\sin (\pi \tilde{y})}{\sinh (\pi \tilde{L} \tilde{x})}\right]
\end{aligned}
$$

See Fig. 1b. Since no summation is present, this algebraic solution does not have a convergence problem. Gebhart [10, p. 98] and Polyanin [11, p. 470] give this expression with the variables interchanged, but no derivation or refer- ence is given; moreover, these references fail to note that this solution is superior for finding the heat flux components. Gebhart [10, p. 101] gives insight into plotting isotherms for Eq. (11). Let the isotherm for the dimensionless temperature be $a$. Setting Eq. (11) equal to $a$, multiplying by $\pi / 2$ and then taking the tangent gives

$\tan \left(a \frac{\pi}{2}\right)=\frac{\sin (\pi \tilde{y})}{\sinh (\pi \widetilde{L} \tilde{x})}$

If $\tilde{y}$ is set equal to a value between zero and one, the corresponding $x$-value is

$\frac{x}{W}=\widetilde{L} \tilde{x}=\frac{1}{\pi} \sinh ^{-1}(\sin (\pi \tilde{y}) / \tan (a \pi / 2))$

which is not given in [10]. Isotherms for $a=0.5,0.1,0.01$, and 0.001 are shown in Fig. 2. A measure of the penetration distance can be found by letting $y / W=1 / 2$ in this equation. This distance is at $x_{\mathrm{p}} / W=1.54,2.27$, and 3.74 for $a=0.01,0.001$, and 0.00001 , respectively. For $a=$ 0.001 , the penetration ratio is about 2.27 , as indicated by Fig. 2. The ratio increases slowly with decreases of $a$ since decreasing $a$ to 0.00001 (a factor of 1/100) increases the penetration distance $x_{\mathrm{p}}$ from 2.27 to 3.74 (only a factor of 1.65). is

For $x=0$, the heat flux in the $x$-direction from Eq. (11)

$$
\begin{aligned}
q_{x, X 10 B 1 Y 11 B 00}(0, y) & =\frac{k T_{0}}{W} \frac{2}{\sin (\pi y / W)} \\
& \approx \frac{k T_{0}}{W} 2\left[\frac{W}{\pi y}+\frac{1}{6} \frac{\pi y}{W}\right], \quad L \rightarrow \infty
\end{aligned}
$$

The approximation is for $y / W \ll 1$. For $y=0$, the heat flux in the $y$-direction is

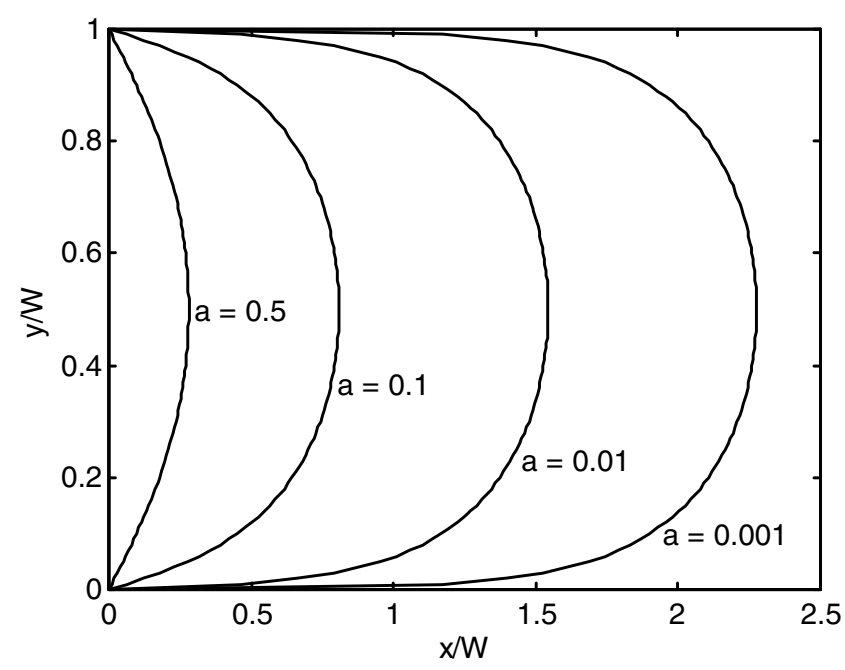

Fig. 2. Isotherms for the $X 10 B 1 Y 11 B 00$ case using Eq. (12b). 


$$
\begin{aligned}
q_{y, X 10 B 1 Y 11 B 00}(x, 0) & =-\frac{k T_{0}}{W} \frac{2}{\sinh (\pi x / W)} \\
& \approx-\frac{k T_{0}}{W} 2\left[\frac{W}{\pi x}-\frac{1}{6} \frac{\pi x}{W}\right], \quad L \rightarrow \infty
\end{aligned}
$$

Again, Eqs. (13a) and (13b) present no convergence problems. Incidentally the corresponding heat fluxes for the quarter-infinite $X 10 B 1 Y 1 B 0$ (Fig. 1d) case are

$q_{x, X 10 B 1 Y 10 B 0}(0, y)=\frac{2}{\pi} \frac{k T_{0}}{y}$,

$q_{y, X 10 B 1 Y 10 B 0}(x, 0)=-\frac{2}{\pi} \frac{k T_{0}}{x}, \quad L$ and $W \rightarrow \infty$

Notice that these heat fluxes are proportional to $1 / x$ and $1 /$ $y$, respectively, and are independent of $W$ and $L$; as a consequence the corresponding heat fluxes become unbounded as $x$ and $y$ go to zero. Also observe that these terms are the first terms in the approximate expressions in Eqs. (13a) and (13b). The heat fluxes can be computed to within about $1 \%$ accuracy using Eq. (14a) for $y / W<0.075$ and using Eq. (14b) for $x / W<0.075$.

\subsection{Finite body solution}

The identity given by Eq. (A.1) is used to improve the convergence of Eq. (9a).

The slowly convergent term is added and subtracted in Eq. (9a) to get

$$
\frac{T(\tilde{x}, \tilde{y})}{T_{0}}=4 \sum_{n=1}^{\infty} \frac{\sin \left(\eta_{n} \tilde{y}\right)}{\eta_{n}}\left(\mathrm{e}^{-\eta_{n} \widetilde{L} \tilde{x}}-\mathrm{e}^{-\eta_{n} \widetilde{L} \tilde{x}}+\frac{\mathrm{e}^{-\eta_{n} \tilde{L} \tilde{x}}-\mathrm{e}^{-\eta_{n} \widetilde{L}(2-\tilde{x})}}{1-\mathrm{e}^{-2 \eta_{n} \tilde{L}}}\right)
$$$$
\eta_{n}=(2 n-1) \pi
$$

Using Eq. (11) and incorporating $-\exp \left(-\eta_{n} x / W\right)$ in the fraction gives the solution denoted $X 11 B 10 Y 11 B 00$ written as

$$
\begin{aligned}
\frac{T(\tilde{x}, \tilde{y})}{T_{0}}= & \frac{2}{\pi} \tan ^{-1}\left[\frac{\sin (\pi \tilde{y})}{\sinh (\pi \widetilde{L} \tilde{x})}\right]+4 \sum_{n=1}^{\infty} \frac{\sin \left(\eta_{n} \tilde{y}\right)}{\eta_{n}} \\
& \times \frac{\mathrm{e}^{-\eta_{n} \widetilde{L}(2+\tilde{x})}-\mathrm{e}^{-\eta_{n} \widetilde{L}(2-\tilde{x})}}{1-\mathrm{e}^{-2 \eta_{n} \widetilde{L}}}
\end{aligned}
$$

Eq. (16) is particularly efficient for $\widetilde{L}$ greater than one. Notice that exponential convergence of the series is present for all values of $x$, unlike Eq. (9a). Eq. (16) also can be modified using an expansion for the denominator given by

$$
\frac{1}{1-\mathrm{e}^{-2 \eta_{n} \widetilde{L}}}=\sum_{m=0}^{\infty} \mathrm{e}^{-2 m \eta_{n} \widetilde{L}}
$$

which yields the full expansion of

$$
\begin{aligned}
\frac{T(\tilde{x}, \tilde{y})}{T_{0}}= & \frac{2}{\pi} \tan ^{-1}\left[\frac{\sin \pi \tilde{y}}{\sinh (\pi \widetilde{L} \tilde{x})}\right] \\
& +\frac{2}{\pi} \sum_{m=1}^{\infty}\left\{\tan ^{-1}\left[\frac{\sin \pi \tilde{y}}{\sinh (\pi \widetilde{L}(2 m+\tilde{x}))}\right]\right. \\
& \left.-\tan ^{-1}\left[\frac{\sin \pi \tilde{y}}{\sinh (\pi \widetilde{L}(2 m-\tilde{x}))}\right]\right\}
\end{aligned}
$$

This expansion can be differentiated with respect to $x$ and $y$ to get exponentially convergent series for the fluxes at the boundaries $x=0$ and $y=0$.

The number of required terms in Eq. (16) is

$$
\begin{aligned}
& \eta_{n_{\max }} \widetilde{L}(2-\tilde{x})=\left(2 n_{\max }\right) \pi \widetilde{L}(2-\tilde{x})=K_{\max } \\
& n_{\max }=\operatorname{ceil}\left(\frac{K_{\max }}{2 \pi \widetilde{L}} \frac{1}{(2-\tilde{x})}\right)
\end{aligned}
$$

Using this equation for $K_{\max }=11.5, \quad \widetilde{L}=0.1$ and $x / L=\tilde{x}=1 / 2$ (or $x / W=0.05$ ), gives a value of 13 . This is a conservative estimate since only 10 terms are needed for this case for absolute errors of less than 0.00001. For larger values of $\widetilde{L}$, fewer terms are needed such as for $\widetilde{L}=1$; in fact only one term in the summation is needed for $\widetilde{L}>K_{\max } /(2 \pi)=11.5 /(2 \pi)=1.83$.

The maximum number of terms in Eq. (17) is about

$m_{\max }=\operatorname{ceil}\left(\frac{K_{\max }}{2 \pi \widetilde{L}}+\frac{\tilde{x}}{2}\right)$

In general, this equation indicates more terms than Eq. (18a), but Eq. (17) might be preferable to Eq. (16) in some cases.

Unlike the slowly convergent equation given by Eq. (9c), using Eq. (16) produces exponentially convergent expressions for heat fluxes. The $x$-direction heat flux is

$$
\begin{aligned}
\frac{q_{x}(x, y)}{k T_{0} / L}= & \widetilde{L}\left\{\frac{2 \cosh (\pi \widetilde{L} \tilde{x}) \sin (\pi \tilde{y})}{\sinh ^{2}(\pi \widetilde{L} \tilde{x})+\sin ^{2}(\pi \tilde{y})}\right. \\
& \left.+4 \sum_{n=1}^{\infty} \sin \left(\eta_{n} \tilde{y}\right) \frac{\mathrm{e}^{-\eta_{n} \tilde{L}(2-\tilde{x})}+\mathrm{e}^{-\eta_{n} \widetilde{L}(2+\tilde{x})}}{1-\mathrm{e}^{-2 \eta_{n} \widetilde{L}}}\right\}
\end{aligned}
$$

The $y$-direction heat flux is

$$
\begin{aligned}
\frac{q_{y}(x, y)}{k T_{0} / L}= & -\widetilde{L}\left\{\frac{2 \sinh (\pi \widetilde{L} \tilde{x}) \cos (\pi \tilde{y})}{\sinh ^{2}(\pi \widetilde{L} \tilde{x})+\sin ^{2}(\pi \tilde{y})}\right. \\
& \left.+4 \sum_{n=1}^{\infty} \cos \left(\eta_{n} \tilde{y}\right) \frac{\mathrm{e}^{-\eta_{n} \tilde{L}(2+\tilde{x})}-\mathrm{e}^{-\eta_{n} \widetilde{L}(2-\tilde{x})}}{1-\mathrm{e}^{-2 \eta_{n} \widetilde{L}}}\right\}
\end{aligned}
$$

Evaluating the first of these at $x=0$ and the second at $y=0$ gives

$$
\begin{aligned}
& \frac{q_{x}(0, \tilde{y})}{k T_{0} / L}=\widetilde{L}\left\{\frac{2}{\sin (\pi \tilde{y})}+8 \sum_{n=1}^{\infty} \sin \left(\eta_{n} \tilde{y}\right) \frac{\mathrm{e}^{-2 \eta_{n} \tilde{L}}}{1-\mathrm{e}^{-2 \eta_{n} \tilde{L}}}\right\}, \\
& \eta_{n}=(2 n-1) \pi
\end{aligned}
$$


$\frac{q_{y}(\tilde{x}, 0)}{k T_{0} / L}=-\widetilde{L}\left\{\frac{2}{\sinh (\pi \tilde{x} \widetilde{L})}+4 \sum_{n=1}^{\infty} \frac{\mathrm{e}^{-\eta_{n} \widetilde{L}(2+\tilde{x})}-\mathrm{e}^{-\eta_{n} \widetilde{L}(2-\tilde{x})}}{1-\mathrm{e}^{-2 \eta_{n} \widetilde{L}}}\right\}$

The heat flux given by Eq. (21a) is less than $1 \%$ in error if the summation is dropped, provided $\widetilde{L} \geqslant 1$. Notice that the first terms on the right hand side in these equations are the same as those given by Eqs. (13a) and (13b) for the $X 10 B 1 Y 11 B 00$ semi-infinite case. Figs. 3 and 4 provide plots of Eqs. (21a) and (21b), respectively. The shapes of the curves are quite different.

Some numerical values for the dimensionless temperature and heat flux components are listed in Table 1a for

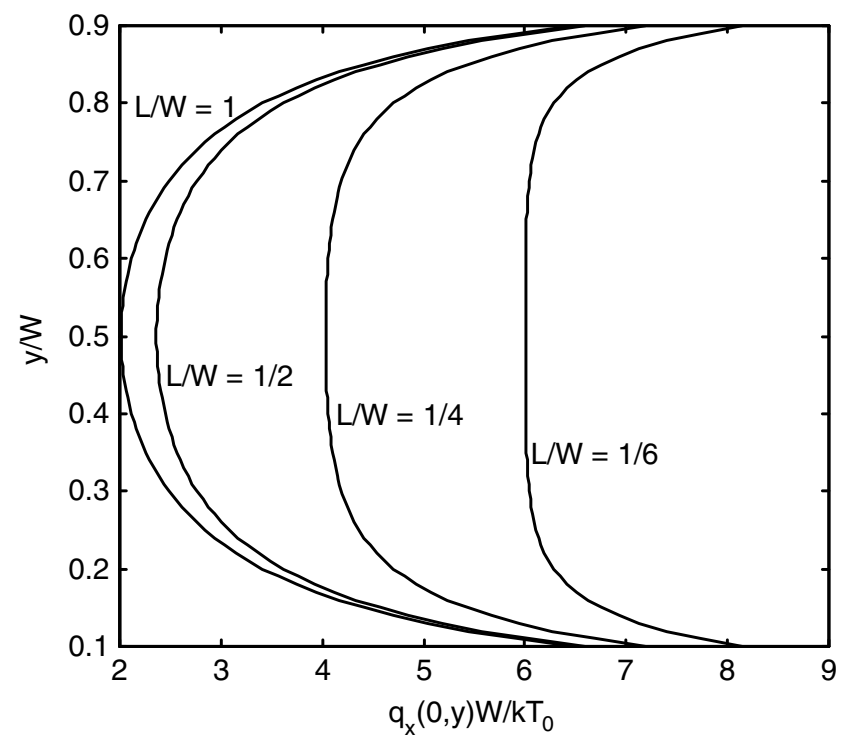

Fig. 3. Dimensionless heat flux given by Eq. (21a) in the $x$-direction at $x=0$ as a function of $y / W$. The $L / W=1$ curve is also valid for $L /$ $W \rightarrow \infty$.

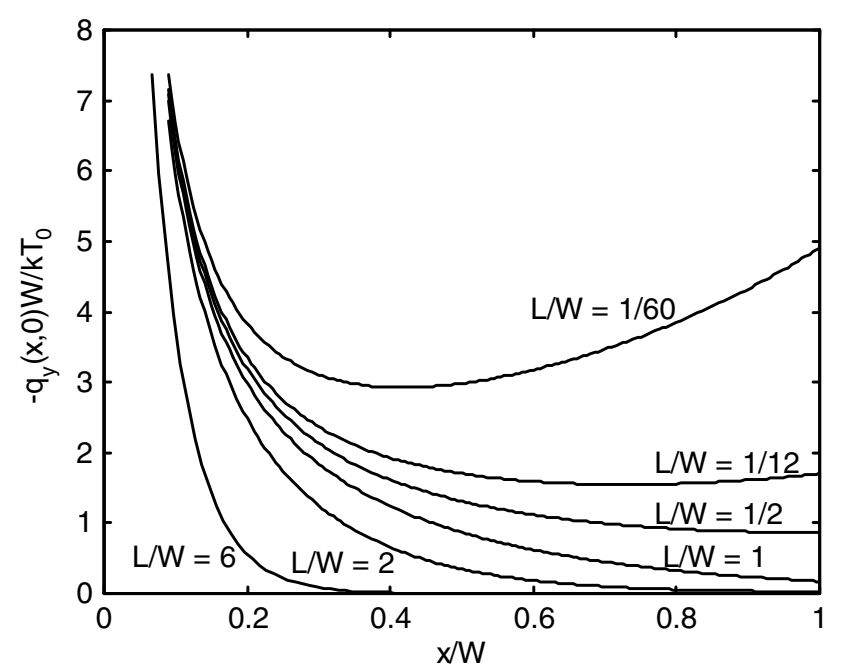

Fig. 4. Negative of the $y$-direction heat flux at $y=0$ for rectangular cases denoted $X 11 B 10 Y 11 B 00$ for various $L / W$ values. $\tilde{x} \equiv x / L=0.5, \tilde{y} \equiv y / W=0.25$ and various values of $\widetilde{L}$. The two $K_{\max }$ values of 11.5 and 23 are used, with the former yielding values with absolute errors of about 0.00001 and the second yielding errors of about $10^{-10}$. The inaccurate digits are indicated by the underlined values and are consistent with the expected accuracy, such as indicated by Eq. (18a). The results show that at most 2 terms in the summation are needed for $\widetilde{L} \geqslant 1$.

\section{Non-standard method (eigenvalues in the non- homogeneous direction)}

For steady conduction in the rectangle, the numerical evaluation of Eq. (7b) can be also improved by reducing the double summation to a single summation with eigenvalues in the non-homogenous direction. This might be called the non-standard method. Re-arrange Eq. (7b) to

$$
T(x, y)=T_{0}\left(\frac{1}{\widetilde{L}}\right)^{2} 2 \sum_{m=1}^{\infty} m \pi \sin (m \pi \tilde{x}) 4 \sum_{n=1}^{\infty} \frac{\sin \left(\eta_{n} \tilde{y}\right)}{\eta_{n}\left[(m \pi / \widetilde{L})^{2}+\eta_{n}^{2}\right]}
$$

Let $m$ be a known quantity in the second summation and use the identity in [8], Eq. (A.1),

$4 \sum_{n=1}^{\infty} \frac{\left[\sin \left(\eta_{n} \tilde{y}\right)\right]}{\eta_{n}\left(C_{m}^{2}+\eta_{n}^{2}\right)}=\frac{1}{C_{m}^{2}}\left[1-\frac{\mathrm{e}^{-C_{m} \tilde{y}}+\mathrm{e}^{-C_{m}(1-\tilde{y})}}{1+\mathrm{e}^{-C_{m}}}\right]$,

$\eta_{n}=(2 n-1) \pi$

Comparing the above two equations reveals that with $C_{m}=m \pi / \widetilde{L}$, the identity can be used to obtain

$T(x, y)=T_{0} 2 \sum_{m=1}^{\infty} \frac{\sin (m \pi \tilde{x})}{m \pi}\left[1-\frac{\mathrm{e}^{-m \pi \tilde{y} / \tilde{L}}+\mathrm{e}^{-m \pi(1-\tilde{y}) / \tilde{L}}}{1+\mathrm{e}^{-m \pi / \tilde{L}}}\right]$

Using the identity (from the $X 11 B 10$ problem)

$2 \sum_{m=1}^{\infty} \frac{\sin (m \pi \tilde{x})}{m \pi}=1-\tilde{x}$

in Eq. (24a) gives the steady-state solution

$$
\begin{aligned}
& \frac{T(\tilde{x}, \tilde{y})}{T_{0}}=(1-\tilde{x})-2 \sum_{m=1}^{\infty} \frac{\sin \left(\beta_{m} \tilde{x}\right)}{\beta_{m}} \frac{\mathrm{e}^{-\beta_{m} \tilde{y} / L}+\mathrm{e}^{-\beta_{m}(1-\tilde{y}) / \tilde{L}}}{1+\mathrm{e}^{-\beta_{m} / \tilde{L}}}, \\
& \beta_{m}=m \pi
\end{aligned}
$$

This equation is analogous to an improved equation [7]. The maximum number of terms analogous to Eqs. (9b) and $(18 \mathrm{a})$ is

$m_{\max }=\operatorname{ceil}\left\{\frac{K_{\max }}{\pi} \max \left(\frac{\widetilde{L}}{\tilde{y}}, \frac{\widetilde{L}}{1-\tilde{y}}\right)\right\}$

Eq. (24c) has exponential convergence everywhere except at $y=0$ and $W$. In particular it converges at all $x$ values, including $x=0$, unlike Eq. (9a). Hence, it is superior to 
Table 1a

Dimensionless temperature and heat flux components for the eigenvalues in the homogeneous direction using Eqs. (16), (19) and (20)

\begin{tabular}{|c|c|c|c|c|c|c|c|}
\hline$x / W$ & $\tilde{y}$ & $\widetilde{L}$ & $K_{\max }$ & $n_{\max }$ & $T(x / W, \tilde{y}) / T_{0}$ & $q_{x}(x, y) L / k T_{0}$ & $q_{y}(x, y) L / k T_{0}$ \\
\hline 0.10 & 0.25 & 0.20 & 11.50 & 7 & $0.487453 \underline{4798}$ & $0.99922 \underline{42348}$ & $-0.039375 \underline{6128}$ \\
\hline 0.10 & 0.25 & 0.20 & 23.00 & 13 & 0.4874535168 & 0.9992238948 & -0.0393751511 \\
\hline 0.25 & 0.25 & 0.50 & 11.50 & 3 & $0.3640566 \underline{550}$ & $0.916991 \underline{3479}$ & $-0.379830 \underline{3110}$ \\
\hline 0.25 & 0.25 & 0.50 & 23.00 & 5 & 0.3640566638 & 0.9169912516 & -0.3798302130 \\
\hline 0.50 & 0.25 & 1.00 & 11.50 & 2 & 0.1820283319 & $0.638795729 \underline{2}$ & $-0.537161038 \underline{5}$ \\
\hline 0.50 & 0.25 & 1.00 & 23.00 & 3 & 0.1820283319 & 0.6387957290 & -0.5371610386 \\
\hline 1.00 & 0.25 & 2.00 & 11.50 & 1 & 0.0388578672 & 0.2453678480 & -0.2435418264 \\
\hline 2.50 & 0.25 & 5.00 & 11.50 & 1 & 0.0003495056 & 0.0054900240 & -0.0054900207 \\
\hline
\end{tabular}

Underlined digits are inaccurate; $K_{\max }=23$ gives values accurate to $10^{-10}$.

Eq. (9a) when temperatures or heat fluxes are needed near $x=0$.

\subsection{Improved non-standard solution}

The convergence of Eq. (24c) can be further improved by removing the poor convergence at $y=0$ and $W$. Add and subtract the slowly convergent term in Eq. (24c) to get

$$
\begin{aligned}
\frac{T(\tilde{x}, \tilde{y})}{T_{0}}= & (1-\tilde{x})-2 \sum_{m=1}^{\infty} \frac{\sin \left(\beta_{m} \tilde{x}\right)}{\beta_{m}}\left[\mathrm{e}^{-\beta_{m} \tilde{y} / \tilde{L}}+\mathrm{e}^{-\beta_{m}(1-\tilde{y}) / \tilde{L}}\right. \\
& \left.-\frac{\mathrm{e}^{-\beta_{m}(1+\tilde{y}) / \tilde{L}}+\mathrm{e}^{-\beta_{m}(2-\tilde{y}) / \tilde{L}}}{1+\mathrm{e}^{-\beta_{m} / L}}\right]
\end{aligned}
$$

Now use the equality given by Eq. (B.3) to find the $X 11 B 10 Y 11 B 00$ solution (best for $\widetilde{L} \ll 1$ ),

$$
\begin{aligned}
\frac{T(\tilde{x}, \tilde{y})}{T_{0}}= & (1-\tilde{x})-\frac{2}{\pi} \tan ^{-1}\left[\frac{\sin (\pi \tilde{x})}{\mathrm{e}^{\pi \tilde{y} / L}-\cos (\pi \tilde{x})}\right] \\
& -\frac{2}{\pi} \tan ^{-1}\left[\frac{\sin (\pi \tilde{x})}{\mathrm{e}^{\pi(1-\tilde{y}) / \tilde{L}}-\cos (\pi \tilde{x})}\right] \\
& +2 \sum_{m=1}^{\infty} \frac{\sin \left(\beta_{m} \tilde{x}\right)}{\beta_{m}} \frac{\mathrm{e}^{-\beta_{m}(1+\tilde{y}) / L}+\mathrm{e}^{-\beta_{m}(2-\tilde{y}) / \tilde{L}}}{1+\mathrm{e}^{-\beta_{m} / \tilde{L}}}, \\
& \beta_{m}=m \pi
\end{aligned}
$$

Notice that the series converges exponentially for all values of $y$. The heat flux components are

$$
\begin{aligned}
\frac{q_{x, X 11 B 10 Y 11 B 00}}{k T_{0} / L}(x, y)= & 1-\frac{\mathrm{e}^{-\pi \tilde{y} / \tilde{L}}-\cos (\pi \tilde{x})}{\cosh (\pi \tilde{y} / \widetilde{L})-\cos (\pi \tilde{x})} \\
& -\frac{\mathrm{e}^{-\pi(1-\tilde{y}) / L}-\cos (\pi \tilde{x})}{\cosh (\pi(1-\tilde{y}) / \widetilde{L})-\cos (\pi \tilde{x})} \\
& -2 \sum_{m=1}^{\infty} \cos \left(\beta_{m} \tilde{x}\right) \\
& \times \frac{\mathrm{e}^{-\beta_{m}(1+\tilde{y}) / \widetilde{L}}+\mathrm{e}^{-\beta_{m}(2-\tilde{y}) / L}}{1+\mathrm{e}^{-\beta_{m} / \widetilde{L}}}
\end{aligned}
$$

$$
\begin{aligned}
\frac{q_{y, X 11 B 10 Y 11 B 00}}{k T_{0} / L}(x, y)= & -\frac{\sin (\pi \tilde{x})}{\cosh (\pi \tilde{y} / \tilde{L})-\cos (\pi \tilde{x})} \\
& +\frac{\sin (\pi \tilde{x})}{\cosh (\pi(1-\tilde{y}) / \widetilde{L})-\cos (\pi \tilde{x})} \\
& -2 \sum_{m=1}^{\infty} \sin \left(\beta_{m} \tilde{x}\right) \\
& \times \frac{-\mathrm{e}^{-\beta_{m}(1+\tilde{y}) / L}+\mathrm{e}^{-\beta_{m}(2-\tilde{y}) / \tilde{L}}}{1+\mathrm{e}^{-\beta_{m} / \tilde{L}}}
\end{aligned}
$$

These equations are particularly efficient for small values of $\widetilde{L}$. A full-expansion equation form for Eq. (26a) is

$$
\begin{aligned}
\frac{T(\tilde{x}, \tilde{y})}{T_{0}}= & (1-\tilde{x})-\frac{2}{\pi} \tan ^{-1}\left[\frac{\sin \pi \tilde{x}}{\mathrm{e}^{\pi \tilde{y} / L}-\cos \pi \tilde{x}}\right] \\
& -\frac{2}{\pi} \sum_{n=1}^{\infty}(-1)^{n}\left\{\tan ^{-1}\left[\frac{\sin \pi \tilde{x}}{\mathrm{e}^{\pi(n+\tilde{y}) / L}-\cos \pi \tilde{x}}\right]\right. \\
& \left.-\tan ^{-1}\left[\frac{\sin \pi \tilde{x}}{\mathrm{e}^{\pi(n-\tilde{y}) / \tilde{L}}-\cos \pi \tilde{x}}\right]\right\}
\end{aligned}
$$

This expansion can be differentiated with respect to $x$ and $y$ to get exponentially convergent series for the fluxes at the boundaries $x=0$ and $y=0$. The maximum number of required terms in each summation in Eqs. (26a)-(26c) is

$m_{\max }=\operatorname{ceil}\left(\frac{K_{\max } \widetilde{L}}{\pi} \max \left(\frac{1}{1+\tilde{y}}, \frac{1}{2-\tilde{y}}\right)\right)$

Numerical values for the dimensionless temperature and heat flux components are listed in Table $1 \mathrm{~b}$ for $\tilde{x}=x /$ $L=0.5, \tilde{y}=0.25$ and various values of $\widetilde{L}$. The results show at most two terms in the summation are needed for $\widetilde{L} \leqslant 0.5$. At most only one term in the summation is needed in Eq. (26a) if $\widetilde{L}$ is less than $\pi / K_{\max }=\pi / 11.5 \approx 0.27$ for $K_{\max }=11.5$, which corresponds to errors about 0.00001 . For such cases, Eq. (26a) reduces to the semi-infinite slab problem, denoted $X 11 B 10 Y 10 B 0$ which has the solution 
Table $1 \mathrm{~b}$

Dimensionless temperature and heat flux components for the eigenvalues in the homogeneous direction using Eqs. (26a)-(26c)

\begin{tabular}{|c|c|c|c|c|c|c|c|}
\hline$x / W$ & $\tilde{y}$ & $\widetilde{L}$ & $K_{\max }$ & $n_{\max }$ & $T(x / W, \tilde{y}) / T_{0}$ & $q_{x}(x, y) L / k T_{0}$ & $q_{y}(x, y) L / k T_{0}$ \\
\hline 0.10 & 0.25 & 0.20 & 11.50 & 1 & 0.4874535168 & 0.9992238948 & -0.0393751511 \\
\hline 0.25 & 0.25 & 0.50 & 11.50 & 2 & 0.3640566638 & 0.9169912516 & -0.3798302129 \\
\hline 0.25 & 0.25 & 0.50 & 23.00 & 3 & 0.3640566638 & 0.9169912516 & -0.3798302130 \\
\hline 0.50 & 0.25 & 1.00 & 11.50 & 3 & $0.182028331 \underline{5}$ & $0.63879 \underline{60309}$ & $-0.5371610 \underline{446}$ \\
\hline 0.50 & 0.25 & 1.00 & 23.00 & 6 & 0.1820283319 & 0.6387957290 & -0.5371610386 \\
\hline 1.00 & 0.25 & 2.00 & 11.50 & 6 & $0.038857 \underline{9638}$ & $0.24536 \underline{81441}$ & $-0.2435 \underline{397294}$ \\
\hline 1.00 & 0.25 & 2.00 & 23.00 & 12 & 0.0388578672 & 0.2453678480 & -0.2435418264 \\
\hline 2.50 & 0.25 & 5.00 & 11.50 & 15 & $0.000349 \underline{4552}$ & $0.00549 \underline{58392}$ & $-0.00549 \underline{26397}$ \\
\hline 2.50 & 0.25 & 5.00 & 23.00 & 30 & 0.0003495056 & 0.0054900240 & -0.0054900207 \\
\hline
\end{tabular}

$\frac{T_{X 11 B 10 Y 10 B 0}(\tilde{x}, \tilde{y})}{T_{0}}=(1-\tilde{x})-\frac{2}{\pi} \tan ^{-1}\left[\frac{\sin (\pi \tilde{x})}{\mathrm{e}^{\pi \tilde{y} / L}-\cos (\pi \tilde{x})}\right]$,

$W \rightarrow \infty$

This solution is for $\widetilde{L} \rightarrow 0$; by also requiring that $\tilde{y}<1 / 2$, the second arc tangent term in Eq. (26a) disappears. The heat flux components associated with this equation are

$$
\begin{aligned}
\frac{q_{x, X 11 B 10 Y 10 B 0}}{k T_{0} / L}(\tilde{x}, \tilde{y}) & =1-\frac{\mathrm{e}^{-\pi \tilde{y} / L}-\cos (\pi \tilde{x})}{\cosh (\pi \tilde{y} / \widetilde{L})-\cos (\pi \tilde{x})} \\
& =\frac{\sinh (\pi \tilde{y} / \widetilde{L})}{\cosh (\pi \tilde{y} / \widetilde{L})-\cos (\pi \tilde{x})}
\end{aligned}
$$

$\frac{q_{y, X 11 B 10 Y 10 B 0}}{k T_{0} / L}(\tilde{x}, \tilde{y})=-\frac{\sin (\pi \tilde{x})}{\cosh (\pi \tilde{y} / \widetilde{L})-\cos (\pi \tilde{x})}$

Evaluating these two equations at $x=0$ and $y=0$, respectively, yields

$$
\begin{aligned}
q_{x, X 11 B 10 Y 10 B 0}(0, y) & =\frac{k T_{0}}{L} \frac{\sinh (\pi \tilde{y} / \widetilde{L})}{\cosh (\pi \tilde{y} / \widetilde{L})-1} \\
& =\frac{k T_{0}}{L} \operatorname{coth}\left(\frac{\pi}{2} \frac{\tilde{y}}{\widetilde{L}}\right) \\
& \approx \frac{k T_{0}}{L}\left(\frac{2}{\pi} \frac{L}{y}+\frac{\pi}{6} \frac{y}{L}\right), \quad W \rightarrow \infty
\end{aligned}
$$

$$
\begin{aligned}
q_{y, X 11 B 10 Y 10 B 0}(x, 0) & =-\frac{k T_{0}}{L} \frac{\sin (\pi \tilde{x})}{1-\cos (\pi \tilde{x})} \\
& =-\frac{k T_{0}}{L} \cot \left(\frac{\pi}{2} \tilde{x}\right) \\
& \approx-\frac{k T_{0}}{L}\left(\frac{2}{\pi} \frac{L}{x}-\frac{\pi}{6} \frac{x}{L}\right), \quad W \rightarrow \infty
\end{aligned}
$$

Notice that the first terms on the right side in Eqs. (28d) and (28e) are exactly the same as for those given by Eqs. (14a) and (14b) for the $X 10 B 1 Y 10 B 0$ case and those by Eqs. (13a) and (13b) for the $X 10 B 1 Y 11 B 00$ case. In addition to showing that the magnitudes of these heat fluxes become unbounded as the origin is approached, the similarity of these equations is an indication of intrinsic verification [3]. See Fig. 1c, which shows this geometry and the temperature solution, Eq. (28a). Also shown are the heat flux equations given by Eqs. (28d) and (28e).

Isotherms for Eq. (28a) can be found by equating it to $a$ and then solving for $y / L$,
$\frac{y}{L}=\frac{1}{\pi} \ln \left[\cos (\pi \tilde{x})+\frac{\sin (\pi \tilde{x})}{\tan [\pi(1-a-\tilde{x}) / 2]}\right]$

Because of the restrictions used in deriving Eq. (29a) from Eq. (28a), all the $x / L$ values from 0 to 1 cannot be used. For $a=0.8$, as shown in Fig. 5 , the $x / L$ range is from 0 to about 0.2 . The deviation in Eq. (28a) from the $1 \mathrm{D}$ solution is

$\delta=\frac{2}{\pi} \tan ^{-1}\left[\frac{\sin (\pi \tilde{x})}{\mathrm{e}^{\pi \tilde{y} / L}-\cos (\pi \tilde{x})}\right]$

Evaluating this equation at $\tilde{x}=1 / 2$ and solving for the $y$ direction penetration distance gives

$\frac{\tilde{y}_{\mathrm{p}}}{\widetilde{L}}=\frac{y_{\mathrm{p}}}{L}=-\frac{1}{\pi} \ln \left(\tan \left(\delta \frac{\pi}{2}\right)\right)$

Numerical values for this penetration distance are $y_{\mathrm{p}} / L=1.32,2.06$ and 3.52 for $\delta=0.01,0.001$ and 0.00001 , respectively. This is for the $X 11 B 10 Y 10 B 0$ case while the other case mentioned below Eq. (12b) is for the body being semi-infinite in the $x$-direction; the numerical values are not greatly different with the values of 1.54 for $a=0.01$ and 1.32 for $\delta=0.01$. It might be helpful to remember that the penetration of the temperature $T$ to about $1 \%$ of its maximum value is an aspect ratio of about 1.5 , which is quite short.

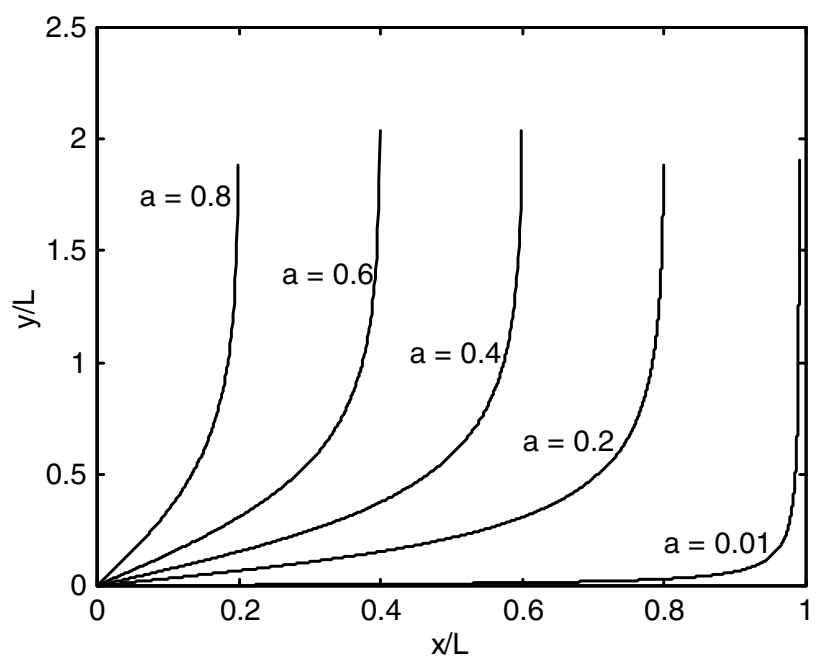

Fig. 5. Isotherms for the $X 11 B 10 Y 10 B 0$ case. 


\section{Steady solution for spatial variation of temperature at the heated surface}

The methods in this paper can be extended in several ways, one of which is to spatial variations of the temperature at $x=0$. Two cases are discussed in this section. The first is for a linear variation in the $y$-direction and the other for a constant temperature from $y=0$ to $W_{1}$ and then zero thereafter. In both cases solutions are given for the eigenvalues in the homogeneous direction, but a partial solution for the second problem is also given with the eigenvalues in the non-homogeneous direction.

\subsection{Linear variation with $y$ at the $x=0$ surface temperature $(X 11 B(y 2) 0 Y 11 B 00)$}

Consider the case of the boundary temperature condition at $x=0$ replaced by

$T(0, y, t)=T_{0} y / W, \quad 0<y<W$

This problem is denoted $X 11 B(y 2) 0 Y 11 B 00$; the " $y 2$ " denotes a linear variation of the temperature at $x=0$. Eq. (5c) becomes

$$
\begin{aligned}
\int_{y^{\prime}=0}^{W} G_{Y 11}\left(y, y^{\prime}, u\right) \frac{y^{\prime}}{W} \mathrm{~d} y^{\prime}= & 2 \sum_{n=1}^{\infty} \\
& \times \frac{\sin (n \pi \tilde{y})(-1)^{n+1}}{n \pi} \mathrm{e}^{-\left(\frac{\eta_{n}}{W}\right)^{2} \alpha u}
\end{aligned}
$$

Similar to Eq. (9a) the steady state temperature is then

$$
\begin{aligned}
T(\tilde{x}, \tilde{y}) & =T_{0} 4 \sum_{m=1}^{\infty} \sum_{n=1}^{\infty} \frac{m \pi \sin (m \pi \tilde{x}) \sin (n \pi \tilde{y})(-1)^{n+1}}{n \pi\left[(m \pi)^{2}+(n \pi \widetilde{L})^{2}\right]} \\
& =T_{0} 2 \sum_{n=1}^{\infty} \frac{\sin (n \pi \tilde{y})(-1)^{n+1}}{n \pi} \frac{\mathrm{e}^{-n \pi \tilde{L} \tilde{x}}-\mathrm{e}^{-n \pi \tilde{L}(2-\tilde{x})}}{1-\mathrm{e}^{-2 n \pi \tilde{L}}}
\end{aligned}
$$

where Eq. (8) is used. However, the single summation does not converge well at $x=0$. The slowly converging term (for small values of $x$ ) is added and subtracted from the second form of Eq. (32) to get,

$$
\begin{aligned}
T(\tilde{x}, \tilde{y})= & T_{0} 2 \sum_{n=1}^{\infty} \frac{\sin (n \pi \tilde{y})(-1)^{n+1}}{n \pi} \\
& \times\left[\mathrm{e}^{-n \pi \tilde{L} \tilde{x}}-\mathrm{e}^{-n \pi \tilde{L} \tilde{x}}+\frac{\mathrm{e}^{-n \pi \tilde{L} \tilde{x}}-\mathrm{e}^{-n \pi \tilde{L}(2-\tilde{x})}}{1-\mathrm{e}^{-2 n \pi \tilde{L}}}\right]
\end{aligned}
$$

Now use the summation identity given by Eq. (B.5) and combine the remaining summations in Eq. (33a) to get

$$
\begin{aligned}
\frac{T(x, y)}{T_{0}}= & \frac{2}{\pi} \tan ^{-1}\left[\frac{\sin (\pi \tilde{y})}{\mathrm{e}^{\pi x / W}+\cos (\pi \tilde{y})}\right] \\
& +2 \sum_{n=1}^{\infty} \frac{\sin (n \pi \tilde{y})(-1)^{n+1}}{n \pi} \frac{\mathrm{e}^{-n \pi \frac{2 L+x}{W}}-\mathrm{e}^{-n \pi \frac{2 L-x}{W}}}{1-\mathrm{e}^{-2 n \pi \tilde{L}}}
\end{aligned}
$$

Notice that the summation disappears for $x=0$ and the arc tangent term yields a linearly increasing temperature equal to the boundary temperature. Also note that the solution simplifies to

$$
\frac{T_{X 10 B(y 2) Y 11 B 00}(x, y)}{T_{0}}=\frac{2}{\pi} \tan ^{-1}\left[\frac{\sin (\pi \tilde{y})}{\mathrm{e}^{\pi x / W}+\cos (\pi \tilde{y})}\right]
$$

for large $\widetilde{L} \equiv L / W$. This case is denoted as $X 10 B(y 2)$ $Y 11 B 00$. This solution has some mathematical similarities with the $X 10 B 1 Y 11 B 00$ case given by Eq. (11).

\subsection{Constant non-zero temperature on part of surface (X11B(y5)0Y11B00)}

The problem with the $x=0$ surface at $T_{0}$ over the region $0<y<W_{1}$ and the rest of the surface at zero is denoted $X 11 B(y 5) 0 Y 11 B 00$, with " $y 5$ " denoting a step change in the surface condition. Convergent solutions for the temperature can be found using the solution given by Eq. (4) with the upper $y^{\prime}$ integration limit changed to $W_{1}$ instead of $W$. Then Eq. (5c) becomes

$$
\begin{aligned}
& \int_{y^{\prime}=0}^{W_{1}} G_{Y 11}\left(y, y^{\prime}, u\right) \mathrm{d} y^{\prime} \\
& \quad=2 \sum_{n=1}^{\infty} \frac{\sin (n \pi \tilde{y})\left[1-\cos \left(n \pi \widetilde{W}_{1}\right)\right]}{n \pi} \mathrm{e}^{-\left(\frac{\eta_{n}}{W}\right)^{2} \alpha u}
\end{aligned}
$$

and analogous to Eq. (9a) the steady state temperature is

$$
\begin{aligned}
T(\tilde{x}, \tilde{y})= & T_{0} 4 \sum_{m=1}^{\infty} \sum_{n=1}^{\infty} \\
& \times \frac{m \pi \sin (m \pi \tilde{x}) \sin (n \pi \tilde{y})\left[1-\cos \left(n \pi \widetilde{W}_{1}\right)\right]}{n \pi\left[(m \pi)^{2}+(n \pi \widetilde{L})^{2}\right]} \\
= & T_{0} 2 \sum_{n=1}^{\infty} \frac{\sin (n \pi \tilde{y})\left[1-\cos \left(n \pi \widetilde{W}_{1}\right)\right]}{n \pi} \\
& \times \frac{\mathrm{e}^{-n \pi \tilde{L} \tilde{x}}-\mathrm{e}^{-n \pi \tilde{L}(2-\tilde{x})}}{1-\mathrm{e}^{-2 n \pi \tilde{L}}}
\end{aligned}
$$

Using the trigonometric identity, $\sin (A) \cos (B)=$ $(\sin (A+B)+\sin (A-B)) / 2$, we can write

$$
\begin{aligned}
& S_{x y}\left(x, y, \widetilde{W}_{1}\right) \\
& =2 \sum_{n=1}^{\infty} \frac{\sin (n \pi \tilde{y})\left[1-\cos \left(n \pi \widetilde{W}_{1}\right)\right]}{n \pi} \mathrm{e}^{-n \pi \tilde{L} \tilde{x}} \\
& =2 \sum_{n=1}^{\infty} \frac{\sin (n \pi \tilde{y})-\frac{1}{2} \sin \left(n \pi\left(\tilde{y}+\widetilde{W}_{1}\right)\right)-\frac{1}{2} \sin \left(n \pi\left(\tilde{y}-\widetilde{W}_{1}\right)\right)}{n \pi} \mathrm{e}^{-n \pi \tilde{L} \tilde{x}}
\end{aligned}
$$

Then using the identity given by Eq. (B.3) yields 


$$
\begin{aligned}
S_{x y}\left(x, y, \widetilde{W}_{1}\right)= & \frac{2}{\pi} \tan ^{-1}\left[\frac{\sin (\pi \tilde{y})}{\mathrm{e}^{\pi L \tilde{x}}-\cos (\pi \tilde{y})}\right] \\
& -\frac{1}{\pi} \tan ^{-1}\left[\frac{\sin \left(\pi\left(\tilde{y}+\widetilde{W}_{1}\right)\right)}{\mathrm{e}^{\pi \widetilde{L}}-\cos \left(\pi\left(\tilde{y}+\widetilde{W}_{1}\right)\right)}\right] \\
& -\frac{1}{\pi} \tan ^{-1}\left[\frac{\sin \left(\pi\left(\tilde{y}-\widetilde{W}_{1}\right)\right)}{\mathrm{e}^{\tilde{L} \tilde{x}}-\cos \left(\pi\left(\tilde{y}-\widetilde{W}_{1}\right)\right)}\right]
\end{aligned}
$$

The final solution is then Eq. (34b) re-written as

$$
\begin{aligned}
\frac{T(x, y)}{T_{0}}= & S_{x y}\left(x, y, \widetilde{W}_{1}\right)+2 \sum_{n=1}^{\infty} \\
& \times \frac{\sin (n \pi \tilde{y})\left[1-\cos \left(n \pi \widetilde{W}_{1}\right)\right]}{n \pi} \\
& \times \frac{\mathrm{e}^{-n \pi \tilde{L}(2+\tilde{x})}-\mathrm{e}^{-n \pi \tilde{L}(2-\tilde{x})}}{1-\mathrm{e}^{-2 n \pi \widetilde{L}}}
\end{aligned}
$$

Analogous comments made below Eq. (33b) also apply. Also Eq. (35c) can be applied to determine an expression for the solution for the region between $W_{1}$ and $W_{2}$ at temperature $T_{0}$ and elsewhere equal to zero. The result is (for $\left.W_{1}<W_{2}\right)$

$$
\begin{aligned}
\frac{T(x, y)}{T_{0}}= & S_{x y}\left(x, y, \widetilde{W}_{2}\right)-S_{x y}\left(x, y, \widetilde{W}_{1}\right) \\
& +2 \sum_{n=1}^{\infty} \frac{\sin (n \pi \tilde{y})\left[\cos \left(n \pi \widetilde{W}_{1}\right)-\cos \left(n \pi \widetilde{W}_{2}\right)\right]}{n \pi} \\
& \times \frac{\mathrm{e}^{-n \pi \widetilde{L}(2+\tilde{x})}-\mathrm{e}^{-n \pi \widetilde{L}(2-\tilde{x})}}{1-\mathrm{e}^{-2 n \pi \tilde{L}}}
\end{aligned}
$$

This equation can be used to approximate any variation of the surface temperature if it is modeled as a series of steps.

The above results for spatial variations are particularly appropriate for large values of $\widetilde{L} \equiv L / W$; they use eigenvalues in the homogeneous direction. Space is not available herein to give complete results for small values of $\widetilde{L}$; however, for small values of $\widetilde{L}$ (or $W / L$ large) and also for $W_{1} /$ $L$ large, the temperature distribution for $y>W_{1}$ is

$\frac{T(x, y)}{T_{0}}=\frac{1}{\pi} \tan ^{-1}\left[\frac{\sin (\pi \tilde{x})}{\mathrm{e}^{\frac{y-W_{1}}{L}}-\cos (\pi \tilde{x})}\right]$

and for $y<W_{1}$ is

$\frac{T(x, y)}{T_{0}}=(1-\tilde{x})-\frac{1}{\pi} \tan ^{-1}\left[\frac{\sin (\pi \tilde{x})}{\mathrm{e}^{\pi \frac{W_{1}-y}{L}}-\cos (\pi \tilde{x})}\right]$

Eqs. (36a) and (36b) can be considered solutions for the $X 11 B(y 5) 0 Y 00$ problem; it might be convenient to visualize the problem as a plate $0<x<L-\infty<y<\infty$. The $x=0$ surface over $-\infty<y^{+}=y-W_{1}<0$ is at $T_{0}$ while the other surfaces are at zero temperature. The origin of the new coordinate $y^{+}$is at $y=W_{1}$. Eq. (36b) has some similarities with Eq. (28a) which is for the $X 11 B 10 Y 10 B 0$ problem.

\section{Comparison of the number of terms in summations}

Several ways are possible to determine the required number of terms in a series. One way is to use the criterion given in this paper in which the exponent is less than $K_{\max }$. This method has at least two advantages. The "for" loop in Matlab can be used instead of the less efficient "if" statement. Also a comparison can be readily made between competing solutions when they contain exponential terms. Before continuing with the comparison of the solutions in this paper, the significance of the criterion is considered.

Consider the similar series in Eqs. (19) and (20); note that the sine and cosine functions are not different in effect in the analysis below. The absolute value of the error in the slower convergent series of Eq. (19) is

$$
\begin{aligned}
\text { |error } \mid & =\left|\sum_{n=n_{\max }+1}^{\infty} \sin ((2 n-1) \pi \tilde{y}) \frac{\mathrm{e}^{-(2 n-1) \tilde{L}(2-\tilde{x})}}{1-\mathrm{e}^{-2(2 n-1) \pi \tilde{L}}}\right| \\
& <\frac{1}{1-\mathrm{e}^{-2\left(2 n_{\max }+1\right) \widetilde{L}}} \sum_{n=n_{\max }+1}^{\infty} \mathrm{e}^{-(2 n-1) \pi \tilde{L}(2-\tilde{x})} \\
& =\frac{1}{1-\mathrm{e}^{-2\left(2 n_{\max }+1\right) \widetilde{L}}} \frac{\mathrm{e}^{\widetilde{\pi L}(2-\tilde{x})} \mathrm{e}^{-2 n_{\max } \pi \widetilde{L}(2-\tilde{x})}}{\mathrm{e}^{2 \widetilde{L}(2-\tilde{x})}-1} \\
& =\frac{1}{1-\mathrm{e}^{-2\left(2 n_{\max }+1\right) \widetilde{L}}} \frac{\mathrm{e}^{-2 n_{\max } \widetilde{L}(2-\tilde{x})}}{2 \sinh [\pi \widetilde{L}(2-\tilde{x})]} \\
& <\frac{\mathrm{e}^{-2 n_{\max } \widetilde{L}(2-\tilde{x})}}{0.99} \text { if } n_{\max } \geqslant 1 \text { and } \widetilde{L} \geqslant \frac{1}{4}
\end{aligned}
$$

Then, the absolute value of the error in the series is less that about $\varepsilon$ if the maximum number of terms in this series in Eq. (19) satisfies

$\mathrm{e}^{-2 n_{\max } \tilde{L}(2-\tilde{x})}=\mathrm{e}^{-K_{\max }}=\varepsilon$

which gives the maximum number of terms equal to

$n_{\max }=\frac{-\ln (\varepsilon)}{2 \pi \widetilde{L}(2-\tilde{x})}=\frac{K_{\max }}{2 \pi \widetilde{L}(2-\tilde{x})}$

This is the same result as given by Eq. (18a), except for its more conservative use of the "ceiling" value. Not only does Eq. (39) give the absolute error, it also gives approximately the relative error for that series. Note that an upper bound of the summation given in Eq. (37) can be found by letting $n_{\max }=0$ to get

$$
\begin{gathered}
\max \left|\sum_{n=1}^{\infty} \sin ((2 n-1) \pi \tilde{y}) \frac{\mathrm{e}^{-(2 n-1) \pi \widetilde{L}(2-\tilde{x})}}{1-\mathrm{e}^{-2(2 n-1) \pi \tilde{L}}}\right| \\
<\frac{1}{1-\mathrm{e}^{-2 \pi \tilde{L}}} \frac{1}{2 \sinh [\pi \widetilde{L}(2-\tilde{x})]} \\
\quad \approx \frac{1}{2 \sinh [\pi \widetilde{L}(2-\tilde{x})]} \text { for } \widetilde{L} \geqslant \frac{1}{4}
\end{gathered}
$$

Comparing this equation with Eq. (37) shows that $\varepsilon$ is also approximately the relative error in the series provided $\widetilde{L}>1 / 4$ which should be satisfied when Eq. (19) is used. 
Eq. (19) has a second term in the series, but it also behaves in the same manner as just described. This analysis can also be extended to the series with $(1+\tilde{y})$ in Eq. (26b). The result is the criterion of

$m_{\max }=\frac{K_{\max } \widetilde{L}}{\pi(1+\tilde{y})}$

where $\widetilde{L}$ can be any value. See Eq. (27) for the condition for both series in Eq. (26b).

A comparison is given of the required number of terms for the four solutions for the $X 11 B 10 Y 11 B 00$ problem. For generality, the equalities are used rather that the ceiling values. Also to avoid specifying values of $K_{\max }$ and $\widetilde{L}$, the homogeneous direction eigenvalue cases use $n_{\max } \widetilde{L} 2 \pi /$ $K_{\max }$ versus $\tilde{x}$. For the standard number of terms given by Eq. (9b), we write

$\frac{n_{\max , \mathrm{std}} 2 \widetilde{L} \pi}{K_{\max }}=\frac{1}{\tilde{x}}$

For the improved equation number of terms given by Eq. (18a), we write

$\frac{n_{\max , \operatorname{imp}} \widetilde{L} 2 \pi}{K_{\max }}=\frac{1}{2-\tilde{x}}$

This criterion is for $T$ obtained from Eq. (16) and the heat fluxes from Eqs. (19) and (20).

For the case of the eigenvalues in the non-homogeneous direction, Eq. (24d) now gives (which we now denote as "standard" to contrast with "improved")

$\frac{m_{\max , \mathrm{std}} 2 \pi}{K_{\max } \widetilde{L}}=2 \max \left(\frac{1}{\tilde{y}}, \frac{1}{1-\tilde{y}}\right)$

The improved (non-homogeneous eigenvalue) case has the normalized number of terms,

$\frac{m_{\max , \operatorname{imp}} 2 \pi}{K_{\max } \widetilde{L}}=2 \max \left(\frac{1}{1+\tilde{y}}, \frac{1}{2-\tilde{y}}\right)$

which comes from Eq. (27) and is used for $T$ and $q$ found from Eqs. (26a)-(26c).

Notice that the definitions of the normalized number of terms are similar, but different in a significant respect relating to the aspect ratio $\widetilde{L} \equiv L / W$. Eqs. (35a) and (35b) need fewer terms as the aspect ratio becomes large while Eqs. (36a) and (36b) indicate that their required numbers of terms are reduced when the aspect ratio becomes small compared with unity.

Plots for Eqs. (42a), (42b) and (43a), (43b) are given in Fig. 6. Scaled values are plotted versus $\tilde{x} \equiv x / L$ for Eqs. (42a) and (42b) and versus $\tilde{y} \equiv y / W$ for Eqs. (43a) and (43b). To promote understanding, it is convenient to imagine that $K_{\max } / 2 \pi=1$ and $\widetilde{L}=1$. Then, the ordinate of the plot is simply the number terms. The improved equation for the number of eigenvalues in the homogeneous direction, $n_{\max } 2 \pi \widetilde{L} / K_{\max }=n_{\max }$ produces the smallest number and the preferred curve. It is the lowest curve in Fig. 6 and is less than one for about all values of $\tilde{x}$ which go from

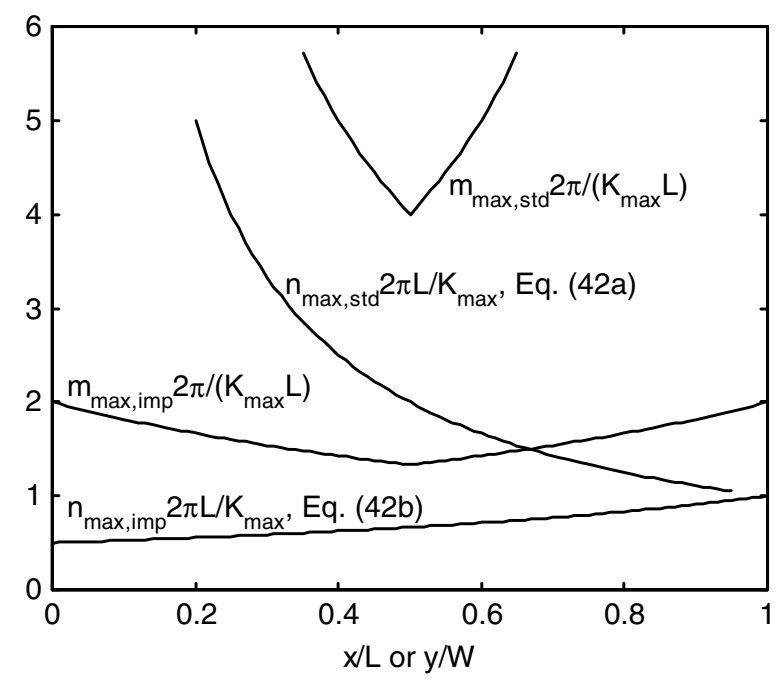

Fig. 6. Scaled number of terms needed in the summations. One scaling is $n_{\max } 2 \pi \widetilde{L} / K_{\max }$ for which the eigenvalues are in the homogeneous direction ( $y$-direction for the $X 11 B 10 Y 11 B 00$ problem) and is plotted versus $\tilde{x} \equiv x / L ; n_{\max }$ is small for large values of the aspect ratio $\widetilde{L}$. The other scaling is $m_{\max } 2 \pi /\left(K_{\max } \widetilde{L}\right)$, which has small values $m_{\max }$ for small $\widetilde{L}$.

0 to 1 . Hence for $1<\widetilde{L}<\infty$ and $K_{\max } / 2 \pi=1$, the summation may not be needed in Eqs. (16), (19), and (20). These conditions give accuracy of about $0.2 \%$ compared to the maximum surface temperature or heat flux at the same $y$ value. On the other hand, for small values of $\widetilde{L}$, such as, $0<\widetilde{L}<0.2$, the $n_{\text {max,imp }}$ values are less than unity and Eqs. (26a)-(26c) may not need the summations.

Whether the final answers are of relative or absolute accuracy needs discussion. The value of a given series is of relative accuracy if its accuracy lies within a given fraction of its true value, which might be small relative to other values. However, if there are two exponential functions, with one subtracted from the other such as in Eq. (26c), the difference may not have this relative accuracy, but the difference of the exponential terms will have the claimed absolute accuracy. Specifically, if $K_{\max }=11.5$, it is expected that the values will be correct to about 0.00001 compared to a maximum value of 1 . For example, see the next to last row and last column of Table $1 \mathrm{~b}$. The dimensionless heat flux of -0.005496 is given, while the correct value is -0.005490 , which are different by 0.000006 . Also note in Eq. (26c), there are other terms besides the series. These terms can be accurately calculated, but if they have about the same and opposite values as the series sum, then the relative accuracy may not be obtained even though the absolute accuracy will be.

\section{Comparison with the $X 11 B 10 Y 11 B 00$ results of Cole and Yen [7]}

Cole and Yen [7] give some results for the same problem in this paper (denoted $X 11 B 10 Y 11 B 00$ ), but with the $x$ - and $y$-directions interchanged. If the standard $x$-direction heat 
Table 2

Comparison of the number of terms with those of ref. [7] for $\widetilde{L}=L / W=1$

\begin{tabular}{lllrrrr}
\hline$\tilde{x}$ & $\tilde{y}$ & $n_{\max }$ & \multicolumn{1}{l}{$\frac{q_{x} L}{k T_{0}}$} & \multicolumn{1}{c}{$\frac{q_{y} L}{k T_{0}}$} & $\begin{array}{l}q_{x}[7] \\
n_{\max }\end{array}$ & $\begin{array}{l}q_{y}[7] \\
n_{\max }\end{array}$ \\
\hline 0.00 & 0.01 & 1 & 63.672921 & 0.000000 & $(580)$ & $(1)$ \\
0.00 & 0.20 & 1 & 3.411401 & 0.000000 & $35,{ }^{*}$ & $1,{ }^{*}$ \\
0.00 & 0.40 & 1 & 2.117159 & 0.000000 & $20,^{*}$ & $1,{ }^{*}$ \\
0.00 & 0.60 & 1 & 2.117159 & 0.000000 & $20,{ }^{*}$ & $1,{ }^{*}$ \\
0.00 & 0.80 & 1 & 3.411401 & 0.000000 & $35,{ }^{*}$ & $1,{ }^{*}$ \\
0.00 & 0.99 & 1 & 63.672921 & 0.000000 & $(580)$ & $(1)$ \\
0.10 & 0.00 & 2 & 0.000000 & -6.257891 & ${ }^{*}, 5$ & $* 65$ \\
0.10 & 0.01 & 2 & 0.640928 & -6.194827 & & \\
0.10 & 0.10 & 2 & 3.290213 & -3.071473 & & \\
0.10 & 0.20 & 2 & 2.767053 & -1.150880 & 35,65 & 35,70 \\
0.10 & 0.40 & 2 & 1.998812 & -0.194620 & 20,70 & 25,75 \\
0.10 & 0.60 & 2 & 1.998812 & 0.194620 & 20,70 & 25,75 \\
0.10 & 0.80 & 2 & 2.767053 & 1.150880 & 35,65 & 35,70 \\
0.10 & 1.00 & 2 & 0.000000 & 6.257891 & ${ }^{*}, 75$ & $* 65$ \\
\hline
\end{tabular}

The last two columns are taken from Ref. [7] except the values inside parentheses, which are estimated to be about what would have been needed.

${ }^{*}$ Series did not converge at boundary.

flux equation (such as those coming from Eq. (9a)) is used, the solution does not converge; see Eq. (10a), which does not converge. In Table 2 of [7], most of the locations for the heat flux require only a moderate number of terms. See Table 2 of the present paper, last two columns. Asterisks denote those solutions that do not converge in [7]. They consider four different solutions, two for $q_{x}$ and two for $q_{y}$. These solutions correspond to those coming from Eqs. (9a) and (24c). In the method presented here, the same number of terms is used for both heat flux components. Conversely in [7] the number of terms in each case is computed separately; the "ratio of the sum of the (absolute values of) the last five terms to the current sum is examined. The series is truncated when this ratio is smaller than $10^{-8}$." The values in [7] are given to six decimal places as shown in Table 2 . The values are computed using $K_{\max }$ $=4 \pi$ for which $\exp \left(-K_{\max }\right)=\exp (-4 \pi)=0.0000035$; this gives answers accurate to six decimal places in this case.

The problem in Table 2 of [7] is for an aspect ratio $\widetilde{L}$ of one; hence, based on the results shown in Fig. 6, the improved equation with the eigenvalues in the homogeneous direction should be used. See the Eq. (35b) curve (the lowest one) in Fig. 6; the number of terms on the ordinate should be doubled for $K_{\max }=4 \pi$, because it is twice the $2 \pi$ in the definition of the dimensionless number of terms. Notice that the numbers of terms in the third column of Table 2 are just 1 or 2 , which is in contrast to the numbers in the last two columns varying from 1 to 70 in [7]. In a certain sense, the difference in computer time between 1 and 70 terms may be insignificant. However, when $\tilde{y}$ is near zero or one, the number of terms can be large. See the extrapolated value of 580 for $\tilde{y}=0.01$ and 0.99 in Table 2. (Parentheses denote values not given in [7].) In contrast, computations based on Eqs. (19) and (20) do not have convergence problems.

\section{Summary and conclusions}

The exact steady-state heat conduction problem for a rectangle with temperature boundary conditions is treated. It is associated with one or more transient problems. The series solutions available in some advanced heat conduction books for the temperature, and particularly the heat flux, converge slowly at the surface where a non-zero temperature is prescribed. Solutions with improved convergence are available [2,4-8]. Even with these latter solutions, further improvement is possible based on a series identity given by Morse and Feshbach [9]. This identity is used in this paper and extended. The solution given in [9] is particularly effective for large aspect ratios in the nonhomogeneous direction, which is the $x$-direction for the $X 11 B 10 Y 11 B 00$ problem. (When the body is semi-infinite in the $x$-direction and $T(0)=T_{0}$, it is denoted as the $X 10 B 1 Y 11 B 00$ problem since $\widetilde{L}=L / W \rightarrow \infty$.) Another algebraic form for a series present in the semi-infinite in the $y$-direction problem is derived in Appendix B. This problem is denoted $X 11 B 10 Y 10 B 0$ and is for the aspect ratio $\widetilde{L}=L / W \rightarrow 0$. These two complementary semi-infinite heat conduction problems are examined in some detail.

The recommended solutions for the temperature are Eqs. (16) and (26a). The former is efficient for $0.8<\widetilde{L}<$ $\infty$ and the latter for $0<\widetilde{L}<0.2$ since then the series components of the temperature and heat fluxes solutions may not be needed. In the mid-range of $0.2<\widetilde{L}<0.8$, two or three terms may be needed, depending upon the accuracy desired. In the limiting cases, the isotherms are relatively easy to obtain and they are displayed in Figs. 2 and 5.

Contrary to common usage, Cole, Yen, Beck, and coworkers $[6-8,21,22]$ have shown that convergence in steady-state problems is frequently improved by using the eigenvalues in direction of the nonhomogeneous boundary conditions, rather than in the homogeneous direction. This statement is generally true. However, this paper shows for temperature boundary conditions, when the algebraic identities can be used, it might not be valid for certain aspect ratios.

Numerical values are given for some cases. An analysis is given to determine the required number of terms in the series to obtain a desired accuracy; the number of terms can be specified in "for" loops in Matlab or "DO" loops in Fortran, providing better computer programming practice.

The methods have many possible extensions and some are given in Section 6. These include prescribed spatial temperature variations at the non-homogenous surface. Two cases are illustrated, one for a linear variation in space and the other for a step change. Others are possible and a basic equation is given for extension to arbitrary prescribed spatial variations.

\section{Acknowledgement}

The insightful and helpful comments of the reviewers are appreciated. 
Appendix A. Derivation of Morse-Feshbach identity [9, p.1179]

The Morse-Feshbach relation [9, p. 1179] can be written as

$$
\begin{aligned}
& \frac{4}{\pi} \sum_{m=1}^{\infty} \mathrm{e}^{-(2 m-1) \pi y / L} \frac{\sin ((2 m-1) \pi x / L)}{2 m-1} \\
& \quad=\frac{2}{\pi} \tan ^{-1}\left[\frac{\sin (\pi x / L)}{\sinh (\pi y / L)}\right]
\end{aligned}
$$

Using complex variables the summation can be expressed by

$$
\begin{aligned}
\frac{4}{\pi} \sum_{m=1}^{\infty} \mathrm{e}^{-(2 m-1) \pi y / L} \frac{\sin ((2 m-1) \pi x / L)}{2 m-1} \\
\quad=\frac{4}{\pi} \sum_{m=1}^{\infty} \mathrm{e}^{-(2 m-1) \pi y / L} \frac{\operatorname{Im}\left[\mathrm{e}^{\mathrm{i}(2 m-1) \pi x / L}\right]}{2 m-1} \\
\quad=\frac{4}{\pi} \operatorname{Im} \sum_{m=1}^{\infty} \frac{\mathrm{e}^{-(2 m-1) \pi\left(\frac{y}{L}-\mathrm{i} \frac{x}{L}\right)}}{2 m-1}=\frac{4}{\pi} \operatorname{Im} \sum_{m=1}^{\infty} \frac{\mathrm{e}^{\mathrm{i}(2 m-1) \pi \frac{x+\mathrm{i} y}{L}}}{2 m-1} \\
\quad=\frac{4}{\pi} \operatorname{Im} \sum_{m=1}^{\infty} \frac{\left(\mathrm{e}^{\left.\mathrm{i} \pi \frac{z}{L}\right)^{2 m-1}}\right.}{2 m-1}=\frac{4}{\pi} \operatorname{Im}\left[\tanh ^{-1} \mathrm{e}^{\left.\mathrm{i} \pi \frac{z}{L}\right]}\right.
\end{aligned}
$$

where [27, Eq. 4.6.33] is used in the last line. Let $w=\tanh ^{-1}(\zeta), \quad \zeta=\mathrm{e}^{\mathrm{i} \pi z / L}$

Then taking the hyperbolic tangent of this equation gives $\tanh (w)=\zeta \quad$ or $\quad \frac{\mathrm{e}^{w}-\mathrm{e}^{-w}}{\mathrm{e}^{w}+\mathrm{e}^{-w}}=\zeta$

Solving for $w$ gives

$w=-\frac{1}{2} \ln \frac{1-\zeta}{1+\zeta}=-\frac{1}{2} \ln \frac{1-\mathrm{e}^{\mathrm{i} \pi z / L}}{1+\mathrm{e}^{\mathrm{i} \pi z / L}}$

Multiply the numerator and denominator inside the logarithm by $\exp (-\mathrm{i} \pi z / 2 L)$ to get

$w=-\frac{1}{2} \ln \frac{\mathrm{e}^{-\mathrm{i} \pi \frac{z}{2 L}}-\mathrm{e}^{\mathrm{i} \pi \frac{z}{2 L}}}{\mathrm{e}^{-\mathrm{i} \pi \frac{z}{2 L}}+\mathrm{e}^{\mathrm{i} \pi \frac{z}{2 L}}}=-\frac{1}{2} \ln \left[\tanh \left(-\mathrm{i} \pi \frac{z}{2 L}\right)\right]$

The hyperbolic tangent of a complex number is [27, Eq. 4.5.51]

$\tanh (z)=\frac{\sinh (2 x)+\mathrm{i} \sin (2 y)}{\cosh (2 x)+\cos (2 y)}$

Then

$$
\begin{aligned}
w & =-\frac{1}{2} \ln \left[\tanh \left(-\mathrm{i} \pi \frac{z}{2 L}\right)\right]=-\frac{1}{2} \ln \left[\tanh \left(\pi \frac{y}{2 L}-\mathrm{i} \pi \frac{x}{2 L}\right)\right] \\
& =-\frac{1}{2} \ln \frac{\sinh (\pi y / L)-\mathrm{i} \sin (\pi x / L)}{\cosh (\pi y / L)+\cos (\pi x / L)}
\end{aligned}
$$

The natural logarithm of a complex number is given by [28, p. 490] as

$$
\ln (z)=\ln |z|+\mathrm{i}\left(\theta_{P}+2 k \pi\right), \quad k=0, \pm 1, \pm 2, \ldots
$$

Then the expression for the imaginary part of $w$ is
$\operatorname{Im} w=-\frac{1}{2} \tan ^{-1}\left(\frac{-\sin \pi x / L}{\sinh \pi y / L}\right)=\frac{1}{2} \tan ^{-1}\left(\frac{\sin \pi x / L}{\sinh \pi y / L}\right)$

This expression leads directly to Eq. (A.1) and a related function given in [9] is

$\frac{4}{\pi} \sum_{m=0}^{\infty} \mathrm{e}^{-(2 m-1) \pi y / L} \frac{\cos ((2 m-1) \pi x / L)}{2 m-1}=\frac{2}{\pi} \tanh ^{-1}\left[\frac{\cos (\pi x / L)}{\cosh (\pi y / L)}\right]$

\section{Appendix B. Derivation of summation identity used in Eq. (26a)}

Consider the summation and utilization of a complex identity to get

$$
\begin{aligned}
2 \sum_{m=1}^{\infty} \frac{\sin (m \pi x / L)}{m \pi} \mathrm{e}^{-m \pi_{L}^{y}} & =2 \sum_{m=1}^{\infty} \frac{\operatorname{Im}\left(\mathrm{e}^{\mathrm{i} m \pi_{L}^{x}}\right)}{m \pi} \mathrm{e}^{-m \pi \frac{y}{L}} \\
& =\frac{2}{\pi} \operatorname{Im} \sum_{m=1}^{\infty} \frac{\mathrm{e}^{-\pi m\left(\frac{y}{L}-\mathrm{i} \frac{x}{L}\right)}}{m}
\end{aligned}
$$

which can also be written in the form (using Mathematica)

$$
\begin{aligned}
\frac{2}{\pi} \operatorname{Im} \sum_{m=1}^{\infty} \frac{\left[\mathrm{e}^{-\pi \frac{y-\mathrm{i} x}{L}}\right]^{m}}{m}= & \frac{2}{\pi} \operatorname{Im}\left[-\ln \left(1-\mathrm{e}^{-\pi \frac{y-\mathrm{i} x}{L}}\right)\right] \\
= & \frac{2}{\pi} \operatorname{Im}\left[-\ln \left(1-\mathrm{e}^{-\pi_{L}^{y}}(\cos (\pi x / L)\right.\right. \\
& +\mathrm{i} \sin (\pi x / L)))]
\end{aligned}
$$

Using Eq. (A.9) then gives the desired identity for $y>0$

$$
\begin{aligned}
2 \sum_{m=1}^{\infty} \frac{\sin (m \pi x / L)}{m \pi} \mathrm{e}^{-m \pi_{L}^{y}} & =-\frac{2}{\pi} \tan ^{-1}\left[\frac{-\mathrm{e}^{-\pi \frac{y}{L}} \sin (\pi x / L)}{1-\mathrm{e}^{-\pi \pi_{L}^{y}} \cos (\pi x / L)}\right] \\
& =\frac{2}{\pi} \tan ^{-1}\left[\frac{\sin (\pi x / L)}{\mathrm{e}^{\pi_{L}^{y}}-\cos (\pi x / L)}\right]
\end{aligned}
$$

Related summation identities are

$\sum_{m=1}^{\infty} \frac{\cos (m \pi x)}{m \pi} \mathrm{e}^{-m \pi y}=-\frac{1}{2 \pi} \ln \left[2 \mathrm{e}^{-\pi y}(\cosh (\pi y)-\cos (\pi x))\right]$,

$y>0$

$\sum_{m=1}^{\infty} \frac{(-1)^{m+1} \sin (m \pi x / L)}{m \pi} \mathrm{e}^{-m \pi y / L}$

$$
=\frac{1}{\pi} \tan ^{-1}\left[\frac{\sin (\pi x / L)}{\mathrm{e}^{\pi y / L}+\cos (\pi x / L)}\right], \quad y>0
$$

\section{References}

[1] P.J. Roach, Verification and Validation in Computational Science and Engineering, Hermosa, Albuquerque, NM, 1998 (Chapters 3-8).

[2] J.V. Beck, A. Haji-Sheikh, D.E. Amos, D.H.Y. Yen, Verification solution for partial heating of rectangular solids, Int. J. Heat Mass Transfer 47 (2004) 4243-4255. 
[3] J.V. Beck, R. McMasters, K.J. Dowding, D.E. Amos, Intrinsic verification methods in linear heat conduction, Int. J. Heat Mass Transfer 49 (2006) 2984-2994.

[4] R.L. McMasters, K. Dowding, J. Beck, D. Yen, Methodology to generate accurate solutions for verification in transient three-dimensional heat conduction, J. Numer. Heat Transfer (B) 41 (6) (2002) 521-541.

[5] J.V. Beck, K.D. Cole, A. Haji-Sheikh, B. Litkouhi, Heat Conduction Using Green's Functions, Hemisphere Publishing Corp., New York, 1992.

[6] P.E. Crittenden, K.D. Cole, Fast-converging steady-state heat conduction in the rectangular parallelepiped, Int. J. Heat Mass Transfer 45 (2002) 3585-3596.

[7] K.D. Cole, D.H.Y. Yen, Green's functions, temperature, and heat flux in the rectangle, Int. J. Heat Mass Transfer 44 (20) (2001) 38833894.

[8] J.V. Beck, K.D. Cole, Improving convergence of summations in heat conduction, Int. J. Heat Mass Transfer 50 (2007) 257-268.

[9] P.M. Morse, H. Feshbach, Methods of Theoretical Physics, McGrawHill, 1953.

[10] B. Gebhart, Heat Conduction and Mass Diffusion, McGraw-Hill, New York, 1993.

[11] A.D. Polyanin, Handbook of Linear Partial Differential Equations for Engineers and Scientists, Chapman and Hall/CRC, Boca Raton, 2002.

[12] L.R. Ingersoll, O.J. Zobel, A.C. Ingersoll, Heat Conduction with Engineering, Geological and Other Applications, The University of Wisconsin Press, Madison, 1954.

[13] Y.A. Melnikov, Green's Functions in Applied Mechanics, Computational Mechanics Publications, Boston, 1995.

[14] Y.A. Melnikov, Influence Functions and Matrices, Marcel Dekker, New York, 1999.
[15] D. Duffy, Green's Functions and Applications, CRC Press, Boca Raton, FL, 2000.

[16] F. de Monte, Transient heat conduction in one-dimensional composite slab, A 'natural analytic' approach, Int. J. Heat Mass Transfer 43 (2000) 3607-3619.

[17] F. de Monte, Unsteady heat conduction in two-dimensional two slabshaped regions, Exact closed form solution and results, Int. J. Heat Mass Transfer 46 (2003) 1455-1469.

[18] L. Yan, A. Haji-Sheikh, J.V. Beck, Thermal characteristics of twolayered bodies with embedded thin-film source, ASME J. Electron. Pack. 115 (1993) 276-283.

[19] A. Haji-Sheikh, J.V. Beck, Temperature solution in multi-layer bodies, Int. J. Heat Mass Transfer 45 (2002) 1865-1877.

[20] C. Aviles-Ramos, A. Haji-Sheikh, J.V. Beck, Exact solutions of heat conduction in composites and application to inverse problems, ASME J. Heat Transfer 120 (1998) 592-599.

[21] K.D. Cole, D.H.Y. Yen, Influence functions for the infinite and semiinfinite strip, J. Thermophys. Heat Transfer 15 (2001) 431-438.

[22] K.D. Cole, Fast-converging series for heat conduction in the circular cylinder, J. Eng. Math. 49 (2004) 217-232.

[23] H.S. Carslaw, J.C. Jaeger, Conduction of Heat in Solids, second ed., Oxford, Oxford, 1959.

[24] V.S. Arpaci, Conduction Heat Transfer, Addison-Wesley, Reading, MA, 1966, p. 194.

[25] G.E. Myers, Analytical Methods in Conduction Heat Transfer, McGraw-Hill, New York, 1971.

[26] M.N. Ozisik, Heat Conduction, second ed., John Wiley, New York, 1993.

[27] M. Abramowitz, I.A. Stegun, Handbook of Mathematical Functions, National Bureau of Standards, Applied Math Series 55, Washington, DC, 1964.

[28] F.B. Hildebrand, Advanced Calculus for Engineers, Prentice-Hall, Englewood Cliffs, NJ, 1948. 